Homology, Homotopy and Applications, vol.4(2), 2002, pp.29-62

\title{
SECONDARY COHOMOLOGY AND THE STEENROD SQUARE
}

\author{
HANS-JOACHIM BAUES
}

(communicated by Larry Lambe)

\begin{abstract}
We introduce and study various properties of the secondary cohomology of a space. Certain Steenrod squares are shown to be related to the action of the symmetric groups on secondary cohomology.
\end{abstract}

\section{To Jan-Erik Roos on his sixty-fifth birthday}

For a field $k$ we choose the Eilenberg-MacLane space $Z^{n}=K(k, n)$ by the realization of the simplicial $k$-vector space generated by the non-basepoint singular simplices of the $n$-sphere $S^{n}=S^{1} \wedge \ldots \wedge S^{1}$. The permutation of the smash product factors $S^{1}$ yields an action of the symmetric group $\sigma_{n}$ on $S^{n}$ and hence on $Z^{n}$. Moreover the quotient map $S^{n} \times S^{m} \rightarrow S^{n+m}$ induces a cup product map $\mu$ : $Z^{n} \times Z^{m} \rightarrow Z^{m+n}$ with $n, m \geqslant 1$; see the Appendix below.

It is well known that the (reduced) cohomology $\widetilde{H}^{n}(X, k)$ of a path-connected pointed space $X$ is the same as the set $\left[X, Z^{n}\right]$ of homotopy classes $\{x\}$ of pointed maps $x: X \rightarrow Z^{n}$. Moreover the cup product of the cohomology algebra $H^{*}=$ $H^{*}(X, k)=\widetilde{H}^{*} \oplus k$ is induced by the map $\mu$, that is $\{x\} \cup\{y\}=\{\mu(x, y)\}$. The cohomology algebra is graded commutative in the sense that

$$
\{x\} \cup\{y\}=(-1)^{n m}\{y\} \cup\{x\}
$$

In this paper we replace the homotopy set $\left[X, Z^{n}\right]$ by the groupoid $\llbracket\left[X, Z^{n} \rrbracket\right.$. The objects of this groupoid are the pointed maps $x: X \rightarrow Z^{n}$ and the morphisms $x \Rightarrow y$ in $\left[\llbracket X, Z^{n}\right]$ are the homotopy classes of homotopies $x \simeq y$ termed tracks. The set of path components of $\llbracket X, Z^{n} \rrbracket$ is

$$
\pi_{0} \llbracket X, Z^{n} \rrbracket=\left[X, Z^{n}\right]=\widetilde{H}^{n}
$$

and the group of tracks $0 \Rightarrow 0$ of the trivial map $0: X \rightarrow * \rightarrow Z^{n}$ in $\llbracket X, Z^{n} \rrbracket$ is

$$
\left.\pi_{1}\left[X, Z^{n}\right]\right]=\left[X, \Omega Z^{n}\right]=\widetilde{H}^{n-1}
$$

We associate with $\llbracket\left[X, Z^{n}\right]$ the exact sequence $\mathcal{H}^{n}(X)$ :

$$
0 \rightarrow \widetilde{H}^{n-1} \rightarrow \mathcal{H}^{n}(X)_{1} \stackrel{\partial}{\longrightarrow} \mathcal{H}^{n}(X)_{0} \rightarrow \widetilde{H}^{n} \rightarrow 0
$$

Here $\mathcal{H}^{n}(X)_{0}$ is the set of all pointed maps $X \rightarrow Z^{n}$ and $\mathcal{H}^{n}(X)_{1}$ is the set of pairs $(x, H)$ with $H: x \Rightarrow 0$ and $\partial(x, H)=x$.

The Eilenberg-MacLane spaces $Z^{n}$ have the following basic properties:

Received November 14, 2000, revised February 14, 2002; published on July 12, 2002.

2000 Mathematics Subject Classification: 55N99.

Key words and phrases: secondary module, secondary cohomology, Hochschild cohomology.

(C) 2002, Hans-Joachim Baues. Permission to copy for private use granted. 
(a) $Z^{n}$ is a $k$-vector space object in the category Top* of pointed spaces.

(b) The symmetric group $\sigma_{n}$ acts on $Z^{n}$ via linear automorphisms inducing the sign of a permutation on $H_{n}\left(Z^{n}\right)$.

(c) The cup product map $\mu: Z^{n} \times Z^{m} \rightarrow Z^{n+m}$ is $k$-bilinear and equivariant with respect to the inclusion $\sigma_{n} \times \sigma_{m} \subset \sigma_{n+m}$. Moreover $\mu$ is associative in the obvious sense and the following diagram commutes.

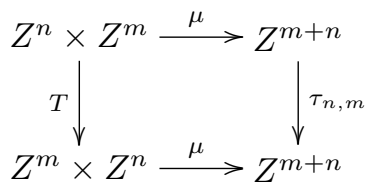

The map $T$ is the interchange map $T(x, y)=(y, x)$ and $\tau_{n, m} \in \sigma_{n+m}$ is the element interchanging the first $n$-block with the second $m$-block.

Properties (b) and (c) imply that $\left\{Z^{n}, n \geqslant 0\right\}$ is a "symmetric spectrum" in the sense of Hovey-Shipley-Smith [HSS] 1.2.5. We use the poperties (a), (b) and (c) of $Z^{n}$ to show that the graded object

$$
\mathcal{H}^{*}(X)=\left\{\mathcal{H}^{n}(X), n \geqslant 1\right\}
$$

has the structure of a "secondary algebra" which we call the secondary cohomology of the space $X$. Using secondary algebras we introduce the third cohomology $\mathrm{SH}^{3}$ of a graded commutative algebra and we show that the secondary cohomology $\mathcal{H}^{*}(X)$ represents an element

$$
\left\langle\mathcal{H}^{*}(X)\right\rangle \in \mathrm{SH}^{3}\left(H^{*}, \widetilde{H}^{*}[1]\right)
$$

which is an invariant of the homotopy type of $X$. There is a natural transformation from the symmetric cohomology $\mathrm{SH}^{3}$ to the Hochschild cohomology $\mathrm{HH}^{3}$ which carries the class $\left\langle\mathcal{H}^{*}(X)\right\rangle$ to the class

$$
\left\langle C^{*}(X)\right\rangle \in \mathrm{HH}^{*}\left(H^{*}, \widetilde{H}^{*}[1]\right)
$$

defined by the algebra of cochains $C^{*}(X)$ of the space $X$. It is known that the class $\left\langle C^{*}(X)\right\rangle$ determines all triple Massey products in the cohomology $H^{*}(X, k)$, see for example Berrick Davydov [BD] or Baues-Minian [BM]. The new class $\left\langle\mathcal{H}^{*}(X)\right\rangle$ in addition determines for $k=\mathbb{F}_{2}$ the Steenrod operations

$$
\mathrm{Sq}^{n-1}: H^{n} \rightarrow H^{2 n-1}, n \geqslant 1 .
$$

The Hochschild cohomology $\mathbf{H H}^{*}$ is defined for algebras and graded algebras in general while the symmetric cohomology $\mathrm{SH}^{3}$ is only defined for commutative graded algebras.

\section{Secondary modules}

Motivated by properties of Eilenberg-MacLane spaces in topology we introduce the algebraic concept of a secondary module. Later we will consider functors from the category of spaces to the categories of secondary modules and secondary algebras respectively. 
Let $k$ be a field and let $R$ be a $k$-algebra with unit $i$ and augmentation $\varepsilon$

$$
k \stackrel{i}{\longrightarrow} R \stackrel{\varepsilon}{\longrightarrow} k .
$$

Here $i$ and $\varepsilon$ are algebra maps with $\varepsilon i=1$. For example let $G$ be a group together with a homomorphism $\varepsilon: G \rightarrow k^{*}$ where $k^{*}$ is the group of units in the field $k$. Then $\varepsilon$ induces an augmentation

$$
\varepsilon: k[G] \rightarrow k
$$

where $k[G]$ is the group algebra of $G$. Here $k[G]$ is a vector space with basis $G$ and $\varepsilon$ carries the basis element $g \in G$ to $\varepsilon(g)$. In particular we have for the symmetric group $\sigma_{n}$ (which is the group of bijections of the set $\{1, \ldots, n\}$ ) the sign-homomorphism

$$
\text { sign : } \sigma_{n} \rightarrow\{1,-1\} \rightarrow k^{*}
$$

which induces the sign-augmentation

$$
\varepsilon=\varepsilon_{\text {sign }}: k\left[\sigma_{n}\right] \rightarrow k
$$

These examples play a special role in applications to topology below.

For $k$-vector spaces $A, B$ we use the tensor product

$$
A \otimes B=A \otimes_{k} B
$$

A homomorphism $f: A \rightarrow B$ is termed a $k$-linear map. If $A$ and $B$ are $R$-modules then the map $f$ is $R$-linear if in addition $f(r \cdot x)=r \cdot f(x)$ for $r \in R, x \in A$. If $R$ and $K$ are $k$-algebras then also $R \otimes K$ is a $k$-algebra with augmentation

$$
\varepsilon: R \otimes K \stackrel{\varepsilon \otimes \varepsilon}{\longrightarrow} k \otimes k=k
$$

The multiplication in $R \otimes K$ is defined as usual by $(\alpha \otimes \beta) \cdot\left(\alpha^{\prime} \otimes \beta^{\prime}\right)=\left(\alpha \alpha^{\prime}\right) \otimes\left(\beta \beta^{\prime}\right)$. Moreover if $X$ is an $R$-module and $Y$ is a $K$-module then $X \otimes Y$ is an $R \otimes K$-module by $(\alpha \otimes \beta) \cdot(x \otimes y)=(\alpha x) \otimes(\beta y)$. The following definition of a secondary module is motivated by the examples in section 3 . Therefore the definition may be considered as a result of calculation derived from these examples, see (2.6) and (2.10). Since, however, secondary modules play a central role in this paper we define them right away as follows.

Definition 1.3. Let $R$ be a $k$-algebra as in (1.1). A secondary module $X=X_{R}$ over $R$ consists of a diagram

$$
R \otimes X_{0} \stackrel{\Gamma}{\longrightarrow} X_{1} \stackrel{\partial}{\longrightarrow} X_{0}
$$

where $X_{0}$ and $X_{1}$ are $R$-modules and $\partial$ is $R$-linear and $\Gamma$ is $k$-linear such that for $r, r^{\prime} \in R, a \in X_{1}, x \in X_{0}$ the following equations hold.

$$
\begin{aligned}
\partial \Gamma(r \otimes x) & =(r-\varepsilon(r)) x \\
\Gamma(r \otimes \partial a) & =(r-\varepsilon(r)) a \\
\Gamma\left(\left(r \cdot r^{\prime}\right) \otimes x\right) & =r \Gamma\left(r^{\prime} \otimes x\right)+\varepsilon\left(r^{\prime}\right) \Gamma(r \otimes x) \\
\Gamma\left(\left(r \cdot r^{\prime}\right) \otimes x\right) & =\Gamma\left(r \otimes r^{\prime} x\right)+\varepsilon(r) \Gamma\left(r^{\prime} \otimes x\right)
\end{aligned}
$$


Now let $X_{R}$ and $Y_{K}$ be secondary modules over $R$ and over $K$ respectively. A map between secondary modules

$$
f=f_{h}: X_{R} \rightarrow Y_{K}
$$

consists of an augmented algebra map $h: R \rightarrow K$ and a commutative diagram

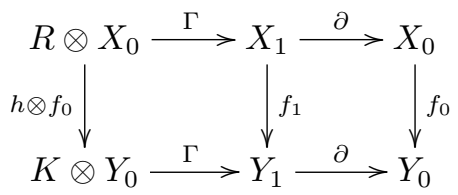

where $f_{1}$ and $f_{0}$ are $k$-linear and $h$-equivariant, i. e. $f_{i}(r \cdot b)=h(r) \cdot f_{i}(b)$ for $r \in R, b \in X_{i}$ and $i=0,1$. Let secmod be the category of secondary modules and let $\operatorname{secmod}(R)$ be the subcategory of secondary modules over $R$ and $R$-equivariant maps $f=f_{h}$ for which $h$ is the identity of $R$.

One readily checks that $\operatorname{secmod}(R)$ is an additive category (in fact an abelian category) with the direct sum $X_{R} \oplus Y_{R}$ given by

$$
R \otimes\left(X_{0} \oplus Y_{0}\right) \stackrel{\Gamma \oplus \Gamma}{\longrightarrow} X_{1} \oplus Y_{1} \stackrel{\partial \oplus \partial}{\longrightarrow} X_{0} \oplus Y_{0}
$$

Moreover for a map $f: X_{R} \rightarrow Y_{R}$ in $\operatorname{secmod}(R)$ the secondary modules $\operatorname{kernel}(f)$ and cokernel $(f)$ are defined in $\operatorname{secmod}(R)$ by using $\operatorname{kernel}\left(f_{i}\right)$ and cokernel $\left(f_{i}\right)$ for $i=0,1$ in the obvious way.

Remark 1.5. Let $R=k[G]$ be a group algebra augmented by $\varepsilon: G \rightarrow k^{*}$ as in (1.1). Then a secondary module $X_{R}$ over $R$ can be identified with a diagram

$$
G \times X_{0} \stackrel{\Gamma}{\longrightarrow} X_{1} \stackrel{\partial}{\longrightarrow} X_{0}
$$

where $X_{1}, X_{0}$ are $k$-vector spaces with an action of $G$ via $k$-linear automorphisms and where $\partial$ is $k$-linear and $G$-equivariant. Moreover $G \times X_{0}$ is the product set and $\Gamma$ is a function between sets which is $k$-linear in $X_{0}$ (i. e. for $g \in G$ the function $X_{0} \rightarrow X_{1}, x \mapsto \Gamma(g, x)$ is $k$-linear). Moreover for $g, g^{\prime} \in G$ the following equations hold.

$$
\begin{aligned}
\partial \Gamma(g, x) & =(g-\varepsilon(g)) x \\
\Gamma(g, \partial a) & =(g-\varepsilon(g)) a \\
\Gamma\left(g g^{\prime}, x\right) & =g \Gamma\left(g^{\prime}, x\right)+\varepsilon\left(g^{\prime}\right) \Gamma(g, x) \\
\Gamma\left(g g^{\prime}, x\right) & =\Gamma\left(g, g^{\prime} x\right)+\varepsilon(g) \Gamma\left(g^{\prime}, x\right)
\end{aligned}
$$

Let $I(R)=\operatorname{kernel}(\varepsilon: R \rightarrow k)$ be the augmentation ideal considered as an $R$ bimodule. For an $R$-module $M$ the tensor product $I(R) \otimes_{R} M$ over $R$ is defined and this tensor product is an $R$-module by $r \cdot(\bar{r} \otimes m)=(r \bar{r}) \otimes m$ for $r \in R, \bar{r} \in I(R)$ and $m \in M$. We have the equation $(\bar{r} \cdot r) \otimes m=\bar{r} \otimes(r \cdot m)$ in $I(R) \otimes_{R} M$.

Lemma 1.6. A secondary $R$-module $X$ can be equivalently described by a commu- 
tative diagram of $R$-linear maps:

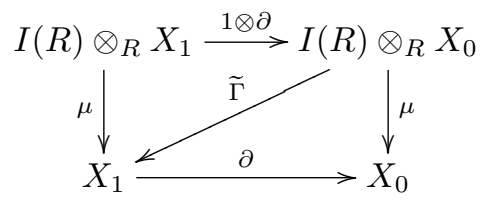

Here $\mu$ is given by $\mu(\bar{r} \otimes m)=\bar{r} \cdot m$ for $m \in X_{1}$ or $m \in X_{0}$.

This characterization of a secondary $R$-module is more appropriate than definition (1.3) which is motivated by topological examples below. In $[\mathbf{B}]$ we consider modules over crossed algebras generalising secondary modules in (1.6).

Proof of (1.6). Given (1.3) we observe that $\Gamma(1 \otimes x)=0$ for $x \in X_{0}$ by (3). Hence $\Gamma$ in (1.3) is determined by the restriction

$$
\Gamma^{\prime}: I(R) \otimes X_{0} \subset R \otimes X_{0} \stackrel{\Gamma}{\longrightarrow} X_{1}
$$

Now (4) shows that $\Gamma^{\prime}$ induces a map

$$
\widetilde{\Gamma}: I(R) \otimes_{R} X_{0} \rightarrow X_{1}
$$

which is $R$-linear by (3). By (1) and (2) we see that the diagram in (1.6) commutes. Conversely given such a diagram we define $\Gamma$ in (1.3) by

$$
\Gamma(r \otimes x)=\widetilde{\Gamma}((r-\varepsilon r) \otimes x)
$$

Now it is easy to show that equations $(1), \ldots,(4)$ are satisfied.

We use (1.6) for the following construction of free secondary $R$-modules.

Definition 1.7. Let $d: V \rightarrow X_{0}$ be an $R$-linear map. Then the free secondary $R$-module $X$ with basis $(V, d)$ is obtained by the following push out in the category of $R$-modules and $R$-linear maps:

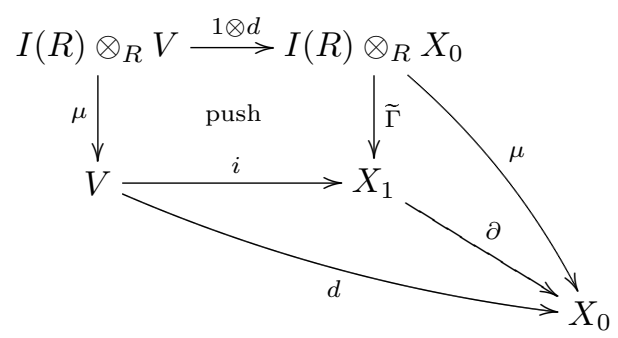

We also write $X_{1}=X_{1}(d)$ and $X=X(d)$. Since $\mu(1 \otimes d)=d \mu$ the $R$-linear map $\partial$ is well defined. Moreover we show that $X$ is a well defined secondary $R$-module: Proof. By (1.6) we have to show that $\widetilde{\Gamma}(1 \otimes \partial)=\mu$ on $I(R) \otimes_{R} X_{1}$. This holds if $\widetilde{\Gamma}(1 \otimes \partial)(1 \otimes i)=\mu(1 \otimes i)$ on $I(R) \otimes_{R} V$ and $\widetilde{\Gamma}(1 \otimes \partial)(1 \otimes \widetilde{\Gamma})=\mu(1 \otimes \widetilde{\Gamma})$ on $I(R) \otimes_{R}\left(I(R) \otimes_{R} X_{0}\right)$. Now the first equation holds since

$$
\widetilde{\Gamma}(1 \otimes \partial)(1 \otimes i)=\widetilde{\Gamma}(1 \otimes(\partial i))=\widetilde{\Gamma}(1 \otimes d)=i \mu=\mu(1 \otimes i)
$$


Here $i \mu=\mu(1 \otimes i)$ holds since $i$ is $R$-linear. For the second equation we get

$$
\widetilde{\Gamma}(1 \otimes \partial)(1 \otimes \widetilde{\Gamma})=\widetilde{\Gamma}(1 \otimes(\partial \widetilde{\Gamma}))=\widetilde{\Gamma}(1 \otimes \mu)=\mu(1 \otimes \widetilde{\Gamma})
$$

Here the last equation holds since for $r, \bar{r} \in I(R), x \in X_{0}$ we have

$$
\begin{aligned}
\widetilde{\Gamma}(1 \otimes \mu)(r \otimes \bar{r} \otimes x) & =\widetilde{\Gamma}(r \otimes(\bar{r} \cdot x)) \\
& =\widetilde{\Gamma}((r \cdot \bar{r}) \otimes x) \\
& =r \widetilde{\Gamma}(\bar{r} \otimes x) \\
& =\mu(1 \otimes \widetilde{\Gamma})(r \otimes \bar{r} \otimes x)
\end{aligned}
$$

Here we use the fact the $\widetilde{\Gamma}$ is $R$-linear.

One readily checks that the free secondary module $X(d)$ has the following universal property: Let $X$ be an object in $\operatorname{secmod}(R)$ and let

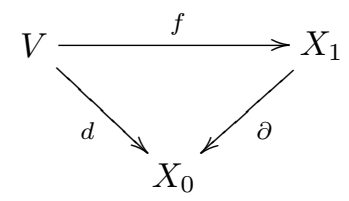

be a commutative diagram of $R$-linear maps. Then there is a unique map $\bar{f}: X(d) \rightarrow$ $X$ in $\operatorname{secmod}(R)$ of the form

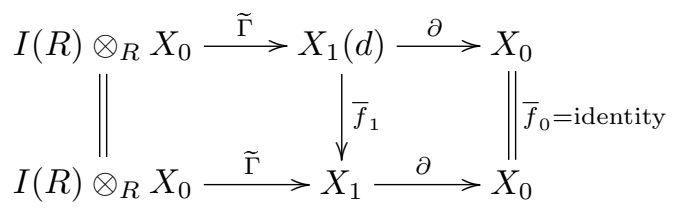

such that $\bar{f}_{1} i=f$ for $i: V \rightarrow X_{1}(d)$ defined in (1.7).

\section{Examples of secondary modules in topology}

We describe examples of secondary modules which arise in topology. Let Top* be the category of pointed topological spaces with base point. This is a groupoid enriched category in the following sense. For objects $X, Y$ in Top* the morphism object $\llbracket X ; Y \rrbracket$ is the groupoid given as follows. Objects in $\llbracket X, Y \rrbracket$ are the pointed maps $X \rightarrow Y$ and for pointed maps $f, g: X \rightarrow Y$ the morphisms $H: f \Rightarrow g$ in $\llbracket X, Y \rrbracket$ are the tracks from $f$ to $g$, that is $H$ is a homotopy class of homotopies $f \simeq g$. The composite of tracks

$$
h \stackrel{G}{\Longleftarrow} g \stackrel{H}{\Longleftarrow} f
$$

is denoted by $G \square H$ where $G \square H$ is defined by adding homotopies in the usual way. The inverse of the track $H$ is denoted by $H^{o p}: g \Rightarrow f$ with $H^{o p} \square H=\widehat{0}_{f}$ where $\widehat{0}_{f}$ denotes the identity track of $f$. 
If $Y \times Z$ is a product in Top* then

$$
\llbracket X, Y \times Z \rrbracket=\llbracket X, Y \rrbracket \times \llbracket X, Z \rrbracket
$$

is a product of groupoids. This shows that for an algebraic object $Y$ in Top* the groupoid $\llbracket X, Y \rrbracket$ is a corresponding algebraic object in the category Grd of (small) groupoids. For example if $Y$ is an abelian group object in Top* (i. e. an abelian topological group) then $\llbracket X, Y \rrbracket$ is a abelian group object in the category Grd. A map between abelian group objects which is a homomorphism of the group structure is termed a linear map.

Let $\mathbf{C}$ be a category. Then the category of pairs in $\mathbf{C}$ denoted by $\operatorname{pair}(\mathbf{C})$ is defined. Objects are morphisms $f: A \rightarrow B$ in $\mathbf{C}$ and morphisms $(\alpha, \beta): f \rightarrow g$ in pair $(\mathbf{C})$ are commutative diagrams in $\mathbf{C}$

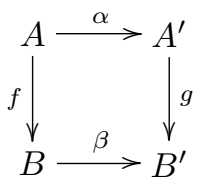

Let $\mathbf{A b}$ be the category of abelian groups. The following result is well known.

Proposition 2.2. The category of abelian group objects in $\mathbf{G r d}$ and linear maps is equivalent to the category $\operatorname{pair}(\mathbf{A b})$.

In order to fix notation we give a proof of this result. Given an abelian group object $G$ in Grd we obtain the object

$$
\partial: G_{1}^{0} \rightarrow G_{0}
$$

in pair $(\mathbf{A b})$ as follows. Here $G_{0}$ is the set of objects of $G$ which is an abelian group since $G$ is an abelian group object in Grd. Let $0 \in G_{0}$ be the neutral object in the abelian group $G_{0}$. Then $G_{1}^{0}$ is the set of all morphisms $f: a \Rightarrow 0$ in $G$ with $a \in G_{0}$ and $\partial f=a$. The abelian group structure of $G_{1}^{0}$ is defined by

$$
(f: a \Rightarrow 0)+(g: b \Rightarrow 0)=(f+g: a+b \rightarrow 0+0=0)
$$

where the right hand side is defined since $G$ is an abelian group object in Grd.

Conversely given an object $\partial: A_{1} \rightarrow A_{0}$ in pair $(\mathbf{A b})$ we define the abelian group object $G(\partial)$ in Grd as follows. The set of objects of $G(\partial)$ is the set $A_{0}$. The set of morphisms of $G(\partial)$ is the product set $A_{1} \times A_{0}$ where $\left(a_{1}, x\right) \in A_{1} \times A_{0}$ is a morphism $\left(a_{1}, x\right): \partial a_{1}+x \rightarrow x$ in $G(\partial)$ also denoted by $\left(a_{1}, x\right)=a_{1}+x$. The identity of $x$ is $(0, x): x=\partial 0+x \rightarrow x$. Composition of

$$
1 x \stackrel{\left(a_{1}, x\right)}{\longleftarrow} \partial a_{1}+x \stackrel{\left(b_{1}, \partial a_{1}+x\right)}{\longleftarrow} \partial b_{1}+\partial a_{1}+x
$$

is $\left(b_{1}+a_{1}, x\right)$ for $a_{1}, b_{1} \in A_{1}$ and $x \in A_{0}$. Now it is readily seen that this way one gets an equivalence of categories.

There are well known generalizations of (2.2). In particular the category of unital groups in Grd is equivalent to the category of crossed modules in the sense of J.H.C. Whitehead, see for example Porter $[\mathbf{P}]$. 
Now given an abelian group object $Y$ in Top* the abelian group object $G=$ $\llbracket X, Y \rrbracket$ in Grd is given via (2.2) by a homomorphism

$$
G_{1}^{0}=\llbracket X, Y \rrbracket_{1}^{0} \stackrel{\partial}{\longrightarrow} G_{0}=\llbracket X, Y \rrbracket_{0}
$$

where $G_{0}$ is the set of all pointed maps $f: X \rightarrow Y$ and where $G_{1}^{0}$ is the set of all tracks $H: f \Rightarrow 0$ with $f \in G_{0}$ and $\partial H=f$. The group structure of $Y$ induces the group structure on $G_{0}$ and $G_{1}^{0}$ in the obvious way.

Definition 2.4. Let $R$ be a $k$-algebra with augmentation $\varepsilon: R \rightarrow k$ as in (1.1). A topological track module $Y$ over $R$ is a $R$-module object $Y$ in Top* (i. e. a topological $R$-module) for which each map $r: Y \rightarrow Y$ given by $r \in R$ admits a unique track $\Gamma_{r}: r \Rightarrow \varepsilon r$ where $\varepsilon r: Y \rightarrow Y$ is defined by the $k$-vector space structure of $Y$.

Example 2.5. Let $Z^{n}=K(k, n)$ be an Eilenberg-MacLane space of the underlying abelian group of the field $k$ with the properties in the introduction, see Appendix A. This shows that for $R=k\left[\sigma_{n}\right]$ the space $Z^{n}$ is a topological $R$-module, in fact, a topological track module over $R$ since for $r \in R$ there is a unique track $r \Rightarrow \varepsilon r$ (with $r, \varepsilon r: Z^{n} \rightarrow Z^{n}$ ). Here $\varepsilon$ is the sign-augmentation as in (1.1)(3).

Proposition 2.6. Let $Y$ be a topological track module over $R$. Then for each $X$ in Top* one obtains canonically a secondary module over $R$

$$
R \otimes G_{0} \stackrel{\Gamma}{\longrightarrow} G_{1}^{0} \stackrel{\partial}{\longrightarrow} G_{0}
$$

where $\partial$ is given by the groupoid $G=\llbracket X, Y \rrbracket$ as in (2.3) and where $\Gamma$ is defined by the composite

$$
\Gamma(r \otimes f)=\Gamma_{r-\varepsilon r} f
$$

Here the track $\Gamma_{r-\varepsilon r}: r-\varepsilon r \Rightarrow 0$ is given for $r-\varepsilon r \in R$ by (2.4).

Using (2.6) we obtain for each track module $Y$ over $R$ a functor

$$
\mathcal{H}(-; Y): \text { Top }^{*} \rightarrow \operatorname{secmod}(R)
$$

which carries $X$ to the track module $\mathcal{H}(X ; Y)=\left(G_{1}^{0}, G_{0}, \partial, \Gamma\right)$ given by $\llbracket X, Y \rrbracket$ in (2.6). Of course we have

$$
\begin{aligned}
& \pi_{0} \mathcal{H}(X, Y)=\operatorname{cokernel}(\partial)=[X, Y] \\
& \pi_{1} \mathcal{H}(X, Y)=\operatorname{kernel}(\partial)=[X, \Omega Y]
\end{aligned}
$$

Here $[X, Y]$ denotes the set of homotopy classes of pointed maps $X \rightarrow Y$ and $\Omega Y$ is the loop space of $Y$. The elements $H \in[X, \Omega Y]$ are identified with the tracks $H: 0 \Rightarrow 0$ where $0: X \rightarrow * \rightarrow Y$ is the zero map. By (1) and (2) we see that the functor (2.7) carries homotopy equivalences in Top* to weak equivalences between secondary modules as defined in the next section.

We are mainly interested in the secondary module

$$
\mathcal{H}^{n}(X)=\mathcal{H}\left(X, Z^{n}\right)
$$

over $R_{n}=k\left[\sigma_{n}\right]$ given by $(2.5)$ with $\pi_{0} \mathcal{H}^{n}(X)=\widetilde{H}^{n}(X, k)$ and $\pi_{1} \mathcal{H}^{n}(X)=$ $\widetilde{H}^{n-1}(X, k)$. This corresponds to the boundary map $\partial$ in the introduction. 
Proof of (2.6). By definition of $\partial$ we have $\partial\left(\Gamma_{r-\varepsilon r} f\right)=(r-\varepsilon r) f$ so that $(1.3)(1)$ is satisfied. Now let $H: f \Rightarrow 0$ be an element in $G_{1}$ with $\partial H=f$. Then we get

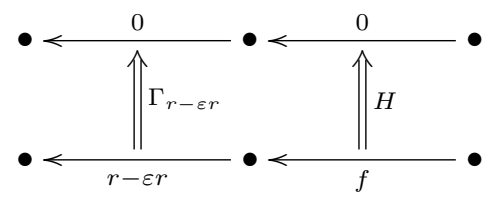

so that for $\Gamma=\Gamma_{r-\varepsilon r}$

$$
\begin{aligned}
\Gamma * H & =0 H \square \Gamma f=\Gamma 0 \square(r-\varepsilon r) H \\
& =\widehat{0}_{0} \square \Gamma f=\widehat{0}_{0} \square(r-\varepsilon r) H \\
& =\Gamma f=(r-\varepsilon r) H
\end{aligned}
$$

and this implies (1.3)(2). Next we have by uniqueness of tracks in $\llbracket Y, Y \rrbracket$ the equations

$$
\begin{aligned}
\Gamma_{r r^{\prime}-\varepsilon\left(r r^{\prime}\right)} & =r \Gamma_{r-\varepsilon r^{\prime}}+\varepsilon\left(r^{\prime}\right) \Gamma_{r-\varepsilon r} \\
& =\Gamma_{r-\varepsilon r} r^{\prime}+\varepsilon(r) \Gamma_{r^{\prime}-\varepsilon r^{\prime}}
\end{aligned}
$$

and these equations imply $(1.3)(3),(4)$.

Remark 2.9. Let $Y$ be given as in (2.6) and let $\widehat{R} \subset \llbracket Y, Y \rrbracket$ be the full subgroupoid with objects given by maps $r: Y \rightarrow Y$ for $r \in R$. Then we obtain the action

$$
\mu_{R}: \widehat{R} \times \llbracket X, Y \rrbracket \subset \llbracket Y, Y \rrbracket \times \llbracket X, Y \rrbracket \stackrel{\circ}{\longrightarrow} \llbracket X, Y \rrbracket
$$

where the second arrow is composition in the groupoid enriched category Top*. The action $\mu_{R}$ determines $\Gamma$ in the secondary module $\mathcal{H}(X, Y)$ given by $(2.6)$ and conversely $\mathcal{H}(X, Y)$ determines uniquely the action $\mu_{R}$. In this sense a secondary module is a $\widehat{R}$-module in the category Grd of groupoids. Here $\widehat{R}$ is the groupoid with objects $R$, path components $\varepsilon^{-1}(x)$ with $x \in k$ and all automorphism groups in $\widehat{R}$ are trivial. The algebra structure of $R$ yields a corresponding structure of $\widehat{R}$.

As pointed out by the referee this remark corresponds to the followoing result generalizing (2.2).

Proposition 2.10. Let $\widehat{R}$ be the internal $k$-algebra in the category of groupoids Grd given by $R$ similarly as in (2.9). Then the category of $\widehat{R}$-internal modules in Grd is equivalent to the category $\operatorname{secmod}(R)$ of secondary modules over $R$.

The proof of (2.10) uses similar arguments to the proof of (2.6). We leave details to the reader. A generalization of $(2.10)$ is proved in [B].

\section{Weak equivalences}

We can consider a secondary module $X$ as a chain complex of $k$-vector spaces concentrated in degree 0 and 1 . The homology of this chain complex is denoted by

$$
\begin{aligned}
& \pi_{0}(X)=\operatorname{cokernel}\left(\partial: X_{1} \rightarrow X_{0}\right) \\
& \pi_{1}(X)=\operatorname{kernel}\left(\partial: X_{1} \rightarrow X_{0}\right)
\end{aligned}
$$


A map $F=F_{h}: X_{R} \rightarrow Y_{K}$ between secondary modules is a weak equivalence if $h: R \rightarrow K$ is an isomorphism and $f$ induces isomorphisms

$$
\begin{aligned}
& f_{*}: \pi_{0}\left(X_{R}\right) \cong \pi_{0}\left(Y_{K}\right) \\
& f_{*}: \pi_{1}\left(X_{R}\right) \cong \pi_{1}\left(Y_{K}\right)
\end{aligned}
$$

We point out that $\pi_{0}(X)$ and $\pi_{1}(X)$ are also $R$-modules for which, however, by (1.3)(1),(2) the $R$-module structure is induced by the augmentation $\varepsilon$, that is $r \cdot x=$ $\varepsilon(r) \cdot x$ for $r \in R, x \in \pi_{0}(X), \pi_{1}(X)$. Hence $\pi_{0}(X)$ and $\pi_{1}(X)$ are just $k$-vector spaces with an action of $R$ via $\varepsilon$. Such $R$-modules are termed $\varepsilon-$ modules. If $X_{R}$ is a secondary module with $\Gamma=0$ then $X_{0}$ and $X_{1}$ are also $\varepsilon$-modules. Hence in this case $X_{R}$ is given by a chain complex $\partial: X_{1} \rightarrow X_{0}$ of $k$-vector spaces. We say that $X_{R}$ is of trivial type if $\Gamma=0$ and $\partial=0$ so that in this case $X_{0}=\pi_{0}$ and $X_{1}=\pi_{1}$.

Two secondary modules $X_{R}, Y_{R}$ are weakly equivalent if there exists a chain of $R$-equivariant weak equivalences

$$
X_{R} \stackrel{\sim}{\sim} X_{1} \stackrel{\sim}{\longrightarrow} X_{2} \stackrel{\sim}{\longleftarrow} \ldots X_{n} \stackrel{\sim}{\longrightarrow} Y_{R} .
$$

Proposition 3.2. Each secondary module is weakly equivalent to a secondary module of trivial type.

We prove this in (3) below. Hence the only invariants of the weak equivalence class of a secondary module $X$ are $\pi_{0} X$ and $\pi_{1} X$.

Remark 3.3. For the secondary module $\mathcal{H}^{n}(X)$ over $R_{n}=k\left[\sigma_{n}\right]$ in (2.7) we know by (3.2) that the weak equivalence type of $\mathcal{H}^{n}(X)$ is trivial. This can also be seen by the following topological argument. By Baues $[\mathbf{B}]$ there exists a sequence

$$
Z^{n} \stackrel{f}{\longleftarrow} Y_{1} \stackrel{g}{\longrightarrow} Y_{2} \stackrel{h}{\longleftarrow} Y_{3}
$$

of topological $R_{n}$-modules and $R_{n}$-linear maps $f, g, h$ with the following properties. The action of $R_{n}$ an $Y_{3}$ satisfies $r \cdot y=\varepsilon(r) \cdot y$ for $r \in R_{n}$ and $y \in Y_{3}$ where $\varepsilon$ is the sign augmentation of $R_{n}$. Moreover $f, g$ and $h$ are homotopy equivalences on Top*. Hence we obtain weak equivalences of secondary modules over $R_{n}$

$$
\mathcal{H}^{n}(X)=\mathcal{H}\left(X, Z^{n}\right) \stackrel{\sim}{\sim} \mathcal{H}\left(X ; Y_{1}\right) \stackrel{\sim}{\longrightarrow} \mathcal{H}\left(X, Y_{2}\right) \stackrel{\sim}{\sim} \mathcal{H}\left(X, Y_{3}\right)
$$

where $\mathcal{H}\left(X, Y_{3}\right)$ is easily seen to be weakly equivalent to a secondary module of trivial type.

For the proof of (3.2) in (3) below we need the following pull back construction for secondary modules. Let $X_{R}$ be a secondary module and let $Y_{0}$ be an $R$-module and let $f: Y_{0} \rightarrow X_{0}$ be a $R$-linear map. Then we obtain the following commutative diagram in which the subdiagram 'pull' is a pull back in the category of vector 
spaces.

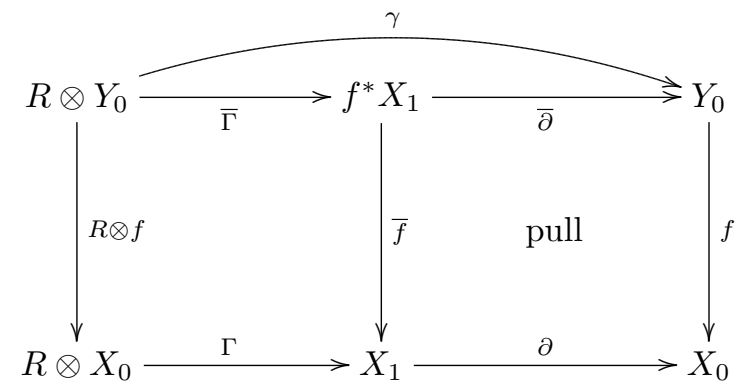

Here $\bar{\Gamma}$ is defined by $\bar{\partial} \bar{\Gamma}=\gamma$ with $\gamma(r \otimes y)=(r-\varepsilon r) \cdot y$ and $\bar{f} \bar{\Gamma}=\Gamma(R \otimes f)$. Then $f^{*} X_{1}$ is an $R$-module and $\bar{\partial}$ is $R$-linear. Moreover we get the following fact.

Lemma 3.4. The top row $Y_{R}=(\bar{\partial}, \bar{\Gamma})=f^{*} X_{R}$ of the diagram is a secondary module over $R$ and $(\bar{f}, f): Y_{R} \rightarrow X_{R}$ is a map in $\operatorname{secmod}(R)$ which is a weak equivalence if $(\partial, f): X_{1} \oplus Y_{0} \rightarrow X_{0}$ is surjective.

The map $f^{*} X_{R} \rightarrow X_{R}$ has the following property. Let $i: K \rightarrow R$ be an augmented map between $k$-algebras and let $g: Z_{K} \rightarrow X_{R}$ be an $i$-equivariant map between secondary modules for which a commutative diagram

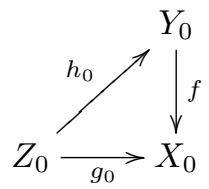

is given. Here $h_{0}$ and $g_{0}$ are $i$-equivariant. Then there exists a unique $i-$ equivariant map $h: Z_{K} \rightarrow f^{*} X_{R}$ for which the diagram

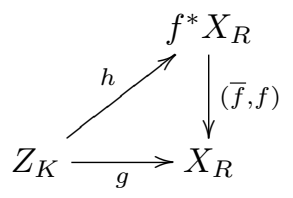

commutes in secmod.

Proof of (3.4). The elements of $f^{*} X_{1}$ are pairs $\left(x_{1}, y\right)$ with $\partial x_{1}=f y$. We define $r\left(x_{1}, y\right)=\left(r x_{1}, r y\right)$ so that $\bar{\partial}$ and $\bar{f}$ are $R$-linear with $\bar{\partial}\left(x_{1}, y\right)=y$ and $\bar{f}\left(x_{1}, y\right)=x_{1}$. Moreover

$$
\bar{\Gamma}(r \otimes y)=(\Gamma(r \otimes f y),(r-\varepsilon r) y)
$$


Hence (1.3)(1) holds for $Y_{R}$. Moreover

$$
\begin{aligned}
\bar{\Gamma}\left(r \otimes \bar{\partial}\left(x_{1}, y\right)\right) & =\bar{\Gamma}(r \otimes y) \\
& =(\Gamma(r \otimes f y),(r-\varepsilon r) y) \\
& =\left(\Gamma\left(r \otimes \partial x_{1}\right),(r-\varepsilon r) y\right) \\
& =\left((r-\varepsilon r) x_{1},(r-\varepsilon r) y\right) \\
& =(r-\varepsilon r)\left(x_{1}, y\right)
\end{aligned}
$$

This shows (1.3)(2) for $Y_{R}$. Next we consider (1.3)(3) for $Y_{R}$ and we get

$$
\begin{aligned}
\bar{\Gamma}\left(r \cdot r^{\prime} \otimes y\right) & =\left(\Gamma\left(r \cdot r^{\prime} \otimes f y\right),\left(r \cdot r^{\prime}-\varepsilon\left(r r^{\prime}\right)\right) y\right) \\
r \bar{\Gamma}\left(r^{\prime} \otimes y\right)+\varepsilon\left(r^{\prime}\right) \bar{\Gamma}(r \otimes y) & =r\left(\Gamma\left(r^{\prime} \otimes f y\right),\left(r^{\prime}-\varepsilon r^{\prime}\right) y\right)+\varepsilon\left(r^{\prime}\right)(\Gamma(r \otimes f y),(r-\varepsilon r) y) \\
& =\left(\Gamma\left(r r^{\prime} \otimes f y\right), r\left(r^{\prime}-\varepsilon r^{\prime}\right) y+\varepsilon\left(r^{\prime}\right)(r-\varepsilon r) y\right) \\
& =\left(\Gamma\left(r r^{\prime} \otimes f g\right),\left(r r^{\prime}-\varepsilon\left(r r^{\prime}\right)\right) y\right) .
\end{aligned}
$$

Similarly one checks (1.3)(4) for $Y_{R}$. Hence $(f, \bar{f}): Y_{R} \rightarrow X_{R}$ is a well defined map between secondary modules. If $(\partial, f)$ is surjective then the pull back is also a push out and therefore $(\bar{f}, f)$ is a weak equivalence.

Now given a secondary module $X_{R}$ we can choose a $k$-linear section $s: \pi_{0} \rightarrow X_{0}$ of the quotient map $q: X_{0} \rightarrow X_{0} / \operatorname{im}(\partial)=\pi_{0}$. Hence the $R$-linear map

$$
f: R \otimes \pi_{0} \rightarrow X_{0}
$$

with $f(r \otimes x)=r \cdot s x$ is defined with $q f(r \otimes x)=\varepsilon(r) \cdot x$. Here $R \otimes \pi_{0}$ is a free $R$-module with the action of $R$ given by $r \cdot\left(r^{\prime} \otimes x\right)=\left(r \cdot r^{\prime}\right) \otimes x$ and $q f$ coincides with the $R$-linear map

$$
q f=\varepsilon \otimes 1: R \otimes \pi_{0} \rightarrow k \otimes \pi_{0}=\pi_{0}
$$

For $I(R)=\operatorname{ker}(\varepsilon: R \rightarrow k)$ we have $\operatorname{ker}(\varepsilon \otimes 1)=I(R) \otimes \pi_{0}$. Using $f$ in (3.5) we get as in (3.4) an $R$-linear map between secondary modules

$$
(\bar{f}, f): Y_{R}=f^{*} X_{R} \rightarrow X_{R}
$$

which is a weak equivalence since $(\partial, f): X_{1} \oplus Y_{0}=X_{1} \oplus R \otimes \pi_{0} \rightarrow X_{0}$ is surjective. Here $Y_{R}$ is a secondary module which is special in the following sense. We say that a secondary module $X_{R}$ is special if for $\pi_{0}=\pi_{0} X$ one has

$$
\left\{\begin{array}{l}
X_{0}=R \otimes \pi_{0} \text { and } \\
\operatorname{im}\left(\partial: X_{1} \rightarrow X_{0}\right)=I(R) \otimes \pi_{0} .
\end{array}\right.
$$

Proposition 3.6. A special secondary $R$-module $X_{R}$ admits an $R$-linear section $t: I(R) \otimes \pi_{0} \rightarrow X_{1}$ of $\partial: X_{1} \rightarrow I(R) \otimes \pi_{0}$.

Proof. We define $t$ by the map

$$
\Gamma: R \otimes X_{0}=R \otimes R \otimes \pi_{0} \rightarrow X_{1},
$$

namely for $r^{\prime} \in I(R)$ and $x \in \pi_{0}$ let

$$
t\left(r^{\prime} \otimes x\right)=\Gamma\left(r^{\prime} \otimes 1 \otimes x\right) .
$$


Then we have $\partial t\left(r^{\prime} \otimes x\right)=\partial \Gamma\left(r^{\prime} \otimes 1 \otimes x\right)=\left(r^{\prime}-\varepsilon r^{\prime}\right)(1 \otimes x)=r^{\prime} \otimes x$ since $\varepsilon\left(r^{\prime}\right)=0$. Moreover $t$ is $R$-linear since for $r \in R$

$$
\begin{aligned}
t\left(r \cdot r^{\prime} \otimes x\right) & =\Gamma\left(r \cdot r^{\prime} \otimes 1 \otimes x\right) \\
& =r \Gamma\left(r^{\prime} \otimes 1 \otimes x\right)+\varepsilon\left(r^{\prime}\right) \Gamma(r \otimes 1 \otimes x) \\
& =r \Gamma\left(r^{\prime} \otimes 1 \otimes x\right) \\
& =r \cdot t\left(r^{\prime} \otimes x\right) .
\end{aligned}
$$

Remark 3.7. A converse of (3.6) is also true. Let $\pi_{0}$ and $\pi_{1}$ be $k$-vector spaces and let

$$
\partial: X_{1} \rightarrow I(R) \otimes \pi_{0}
$$

be a surjective $R$-linear map for which $\pi_{1}=\operatorname{ker}(\partial)$ is an $\varepsilon$-module and let $t$ be an $R$-linear section of $\partial$. Then a special secondary $R$-module $X_{R}$ is defined in terms of $\partial$ and $t$ as follows. Let $X_{0}=R \otimes \pi_{0}$ and let

$$
\Gamma: R \otimes X_{0}=R \otimes R \otimes \pi_{0} \rightarrow X_{1}
$$

be given by $\left(r, r^{\prime} \in R, x \in \pi_{0}\right)$

$$
\Gamma\left(r \otimes r^{\prime} \otimes x\right)=(r-\varepsilon r) t\left(\left(r^{\prime}-\varepsilon r^{\prime}\right) \otimes x\right)+\varepsilon\left(r^{\prime}\right) t((r-\varepsilon r) \otimes x)
$$

Then one can check that $\Gamma$ satisfies all the axioms in (1.3) so that $X_{R}$ is a well defined special secondary module. Hence by (3.6) special secondary modules are up to isomorphism determined by $\pi_{0}$ and $\pi_{1}$ with $X_{0}=R \otimes \pi_{0}$ and $X_{1}=\pi_{1} \oplus I(R) \otimes \pi_{0}$.

Proof of (3.2). Let $X_{R}$ be a secondary module. Then we obtain by (3.5) the special secondary module $Y_{R}=f^{*} X_{R}$ and the weak equivalence $Y_{R} \stackrel{\sim}{\longrightarrow} X_{R}$. Moreover by (3.6) and (3.7) we have $Y_{0}=R \otimes \pi_{0}$ and $Y_{1}=\pi_{1} \oplus I(R) \otimes \pi_{0}$ and

$$
\partial: Y_{1}=\pi_{1} \oplus I(R) \otimes \pi_{0} \rightarrow I(R) \otimes \pi_{0} \subset R \otimes \pi_{0}=Y_{0}
$$

is given by the projection and the inclusion. Now we obtain a weak equivalence $g$ with

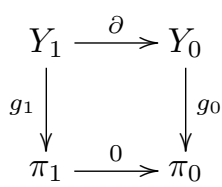

where $g_{0}=\varepsilon \otimes 1$ and $g_{1}$ is the projection. By the definition of $\Gamma$ in (3.7) in terms of the section $t: I(R) \otimes \pi_{0} \subset Y_{1}$ we see that $g_{1} \Gamma=0$ so that $g$ is a well defined map between secondary modules where $0: \pi_{1} \rightarrow \pi_{0}$ is the secondary module of trivial type given by $\pi_{0}$ and $\pi_{1}$.

\section{Tensor products of secondary modules}

Here we introduce the tensor product of secondary modules which is needed for the definition of secondary algebras in the next section. For secondary modules $X_{R}$ 
and $Y_{K}$ the tensor product of the underlying chain complexes is given by the chain complex of $k$-vector spaces

$$
\begin{aligned}
& X_{1} \otimes Y_{1} \stackrel{d_{2}}{\longrightarrow} X_{1} \otimes Y_{0} \oplus X_{0} \otimes Y_{1} \stackrel{d_{1}}{\longrightarrow} X_{0} \otimes Y_{0} \\
& d_{2}(a \otimes b)=(\partial a) \otimes b-a \otimes(\partial b) \\
& d_{1}(a \otimes y)=(\partial a) \otimes y \\
& d_{1}(x \otimes b)=x \otimes(\partial b)
\end{aligned}
$$

with $x \in X_{0}, y \in Y_{0}, a \in X_{1}, b \in Y_{1}$. Hence $d_{1}$ induces the boundary map

$$
\partial_{\otimes}:\left(X_{1} \otimes Y_{0} \oplus X_{0} \otimes Y_{1}\right) / \operatorname{im}\left(d_{2}\right) \rightarrow X_{0} \otimes Y_{0}
$$

Since $k$ is a field we get by the Künneth formula

$$
\begin{aligned}
\pi_{0} \partial_{\otimes} & =\operatorname{cok}\left(\partial_{\otimes}\right)=\pi_{0}(X) \otimes \pi_{0}(Y) \\
\pi_{1} \partial_{\otimes} & =\operatorname{ker}\left(\partial_{\otimes}\right)=\pi_{1}(X) \otimes \pi_{0}(Y) \oplus \pi_{0}(X) \otimes \pi_{1}(Y)
\end{aligned}
$$

One readily checks that $\partial_{\otimes}$ is an $R \otimes K$-equivariant $k$-linear map.

Definition 4.2. We define the tensor product $X_{R} \otimes Y_{K}=(X \otimes Y)_{R \otimes K}$ of secondary modules $X_{R}$ and $Y_{K}$ by the diagram

$$
R \otimes K \otimes X_{0} \otimes Y_{0} \stackrel{\Gamma_{\otimes}}{\longrightarrow}\left(X_{1} \otimes Y_{0} \oplus X_{0} \otimes Y_{1}\right) / i m d_{2} \stackrel{\partial_{\otimes}}{\longrightarrow} X_{0} \otimes Y_{0}
$$

Here $\partial_{\otimes}$ is defined as in (4.1) and $\Gamma_{\otimes}$ is defined by the following formula

$$
\begin{aligned}
\Gamma_{\otimes}(\alpha \otimes \beta \otimes x \otimes y) & =\Gamma(\alpha \otimes x) \otimes(\beta y)+(\varepsilon(\alpha) x) \otimes \Gamma(\beta \otimes y) \\
& =(\alpha x) \otimes \Gamma(\beta \otimes y)+\Gamma(\alpha \otimes x) \otimes(\varepsilon(\beta) y)
\end{aligned}
$$

Here the second equation is a consequence of the first equation since $(\partial a) \otimes b=$ $a \otimes(\partial b)$ by $(4.1)$.

Lemma 4.3. The tensor product $X_{R} \otimes Y_{K}$ of secondary modules $X_{R}$ and $Y_{K}$ is a well defined secondary module over $R \otimes K$.

Proof. The map $\partial_{\otimes}$ is $R \otimes K$-linear and $\Gamma_{\otimes}$ is a well defined $k$-linear map. Hence we have to check the equations (1)... (4) in (1.3): We first check (1).

$$
\begin{aligned}
\partial_{\otimes} \Gamma_{\otimes}(\alpha \otimes \beta \otimes x \otimes y) & =\partial \Gamma(\alpha \otimes x) \otimes \beta y+\varepsilon(\alpha) x \otimes \partial \Gamma(\beta \otimes y) \\
& =(\alpha-\varepsilon(\alpha)) x \otimes \beta y+\varepsilon(\alpha) x \otimes(\beta-\varepsilon(\beta)) y \\
& =\alpha x \otimes \beta y-\varepsilon(\alpha) \varepsilon(\beta) x \otimes y \\
& =(\alpha \otimes \beta-\varepsilon(\alpha \otimes \beta))(x \otimes y)
\end{aligned}
$$

Next we check (2) for $\Gamma_{\otimes}$.

$$
\begin{aligned}
\Gamma_{\otimes}\left(\alpha \otimes \beta \otimes \partial_{\otimes}(a \otimes y)\right) & =\Gamma_{\otimes}(\alpha \otimes \beta \otimes \partial a \otimes y) \\
& =\Gamma(\alpha \otimes \partial a) \otimes \beta y+\varepsilon(\alpha) \partial a \otimes \Gamma(\beta \otimes y) \\
& =(\alpha-\varepsilon(\alpha)) a \otimes \beta y+\varepsilon(\alpha) a \otimes \partial \Gamma(\beta \otimes y), \text { see }(4.1), \\
& =(\alpha-\varepsilon(\alpha)) a \otimes \beta y+\varepsilon(\alpha) a \otimes(\beta-\varepsilon(\beta)) y \\
& =\alpha a \otimes \beta y-\varepsilon(\alpha) \varepsilon(\beta) a \otimes y \\
& =(\alpha \otimes \beta-\varepsilon(\alpha \otimes \beta))(a \otimes y)
\end{aligned}
$$




$$
\begin{aligned}
\Gamma_{\otimes}\left(\alpha \otimes \beta \otimes \partial_{\otimes}(x \otimes b)\right) & =\Gamma_{\otimes}(\alpha \otimes \beta \otimes x \otimes \partial b) \\
& =\Gamma(\alpha \otimes x) \otimes \beta \partial b+\varepsilon(\alpha) x \otimes \Gamma(\beta \otimes \partial b) \\
& =\Gamma(\alpha \otimes x) \otimes \partial(\beta b)+\varepsilon(\alpha) x \otimes(\beta-\varepsilon(\beta)) b \\
& =\partial \Gamma(\alpha \otimes x) \otimes \beta b+\varepsilon(\alpha) x \otimes(\beta-\varepsilon(\beta)) b, \text { see }(4.1), \\
& =(\alpha-\varepsilon(\alpha)) x \otimes \beta b+\varepsilon(\alpha) x \otimes(\beta-\varepsilon(\beta)) b \\
& =\alpha x \otimes \beta b-\varepsilon(\alpha) \varepsilon(\beta) x \otimes b \\
& =(\alpha \otimes \beta-\varepsilon(\alpha \otimes \beta))(x \otimes b)
\end{aligned}
$$

Now we check (3) for $\Gamma_{\otimes}$.

$$
\begin{aligned}
\Gamma_{\otimes}\left((\alpha \otimes \beta)\left(\alpha^{\prime} \otimes \beta^{\prime}\right) \otimes x \otimes y\right) & =\Gamma_{\otimes}\left(\alpha \alpha^{\prime} \otimes \beta \beta^{\prime} \otimes x \otimes y\right) \\
& =\Gamma\left(\alpha \alpha^{\prime} \otimes x\right) \otimes \beta \beta^{\prime} y+\varepsilon\left(\alpha \alpha^{\prime}\right) x \otimes \Gamma\left(\beta \beta^{\prime} \otimes y\right)=(\mathrm{i})
\end{aligned}
$$

Now we get by (3) that (i)=(ii) coincides with

(ii) $=\left(\alpha \Gamma\left(\alpha^{\prime} \otimes x\right)+\varepsilon\left(\alpha^{\prime}\right) \Gamma(\alpha \otimes x)\right) \otimes \beta \beta^{\prime} y+\varepsilon\left(\alpha \alpha^{\prime}\right) x \otimes\left(\beta \Gamma\left(\beta^{\prime} \otimes y\right)+\varepsilon\left(\beta^{\prime}\right) \Gamma(\beta \otimes y)\right)$

On the other hand we have

$$
\begin{aligned}
\text { (iii) }= & (\alpha \otimes \beta) \Gamma_{\otimes}\left(\alpha^{\prime} \otimes \beta^{\prime} \otimes x \otimes y\right)+\varepsilon\left(\alpha^{\prime} \otimes \beta^{\prime}\right) \Gamma_{\otimes}(\alpha \otimes \beta \otimes x \otimes y) \\
= & \alpha \Gamma\left(\alpha^{\prime} \otimes x\right) \otimes \beta \beta^{\prime} y+\varepsilon\left(\alpha^{\prime}\right) \alpha x \otimes \beta \Gamma\left(\beta^{\prime} \otimes y\right) \\
& +\varepsilon\left(\alpha^{\prime} \otimes \beta^{\prime}\right)(\Gamma(\alpha \otimes x) \otimes \beta y+\varepsilon(\alpha) x \otimes \Gamma(\beta \otimes y)
\end{aligned}
$$

We have to check (ii) $=$ (iii). But this is equivalent to

$$
\varepsilon\left(\alpha^{\prime}\right) \Gamma(\alpha \otimes x) \otimes \beta \beta^{\prime} y+\varepsilon\left(\alpha \alpha^{\prime}\right) x \otimes \beta \Gamma\left(\beta^{\prime} \otimes y\right)=\varepsilon\left(\alpha^{\prime}\right) \alpha x \otimes \beta \Gamma\left(\beta^{\prime} \otimes y\right)+\varepsilon\left(\alpha^{\prime} \otimes \beta^{\prime}\right) \Gamma(\alpha \otimes x) \otimes \beta y
$$

This equation is equivalent to

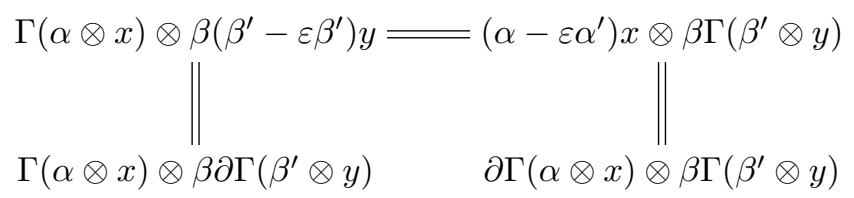

By (4.1) we know that

$$
\begin{aligned}
\Gamma(\alpha \otimes x) \otimes \beta \partial \Gamma\left(\beta^{\prime} \otimes y\right) & =\Gamma(\alpha \otimes x) \otimes \partial \beta \Gamma\left(\beta^{\prime} \otimes y\right) \\
& =\partial \Gamma(\alpha \otimes x) \otimes \beta \Gamma\left(\beta^{\prime} \otimes y\right)
\end{aligned}
$$

This completes the proof that (ii) $=($ iii) and hence (i) $=$ (iii) and hence (3) holds for $X \otimes Y$. Finally we have to check (4). For this we apply (4) to (i) above and we get (i) $=($ iv) where

(iv) $=\left(\Gamma\left(\alpha \otimes \alpha^{\prime} x\right)+\varepsilon(\alpha) \Gamma\left(\alpha^{\prime} \otimes x\right)\right) \otimes \beta \beta^{\prime} y+\varepsilon\left(\alpha \alpha^{\prime}\right) x \otimes\left(\Gamma\left(\beta \otimes \beta^{\prime} y\right)+\varepsilon(\beta) \Gamma\left(\beta^{\prime} \otimes y\right)\right)$

On the other hand we have

$$
\begin{aligned}
(\mathrm{v})= & \Gamma_{\otimes}\left(\alpha \otimes \beta \otimes \alpha^{\prime} x \otimes \beta^{\prime} y\right)+\varepsilon(\alpha \otimes \beta) \Gamma_{\otimes}\left(\alpha^{\prime} \otimes \beta^{\prime} \otimes x \otimes y\right) \\
= & \left.\Gamma\left(\alpha \otimes \alpha^{\prime} x\right) \otimes \beta \beta^{\prime} y+\varepsilon(\alpha) \alpha^{\prime}\right) x \otimes \Gamma\left(\beta \otimes \beta^{\prime} y\right) \\
& +\varepsilon(\alpha \otimes \beta)\left(\Gamma\left(\alpha^{\prime} \otimes x\right) \otimes \beta^{\prime} y+\varepsilon\left(\alpha^{\prime}\right) x \otimes \Gamma\left(\beta^{\prime} \otimes y\right)\right)
\end{aligned}
$$


We have to check (iv) $=(\mathrm{v})$. This is the case if and only if the following equation holds.

$$
\begin{aligned}
& \varepsilon(\alpha) \Gamma\left(\alpha^{\prime} \otimes x\right) \otimes \beta \beta^{\prime} y+\varepsilon\left(\alpha \alpha^{\prime}\right) x \otimes \Gamma\left(\beta \otimes \beta^{\prime} y\right) \\
& =\varepsilon(\alpha) \alpha^{\prime} x \otimes \Gamma\left(\beta \otimes \beta^{\prime} y\right)+\varepsilon(\alpha \otimes \beta) \Gamma\left(\alpha^{\prime} \otimes x\right) \otimes \beta^{\prime} y
\end{aligned}
$$

This equation holds if and only if the following equation is true

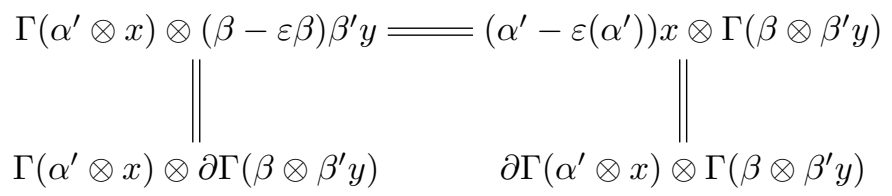

Now again (4.1) shows that this equation is true. Hence we have shown $(\mathrm{i})=(\mathrm{v})$ and this corresponds to equation (4) for $X \otimes Y$. Now the proof of the lemma is complete.

Lemma 4.4. The tensor product of secondary modules is associative and bilinear, that is:

$$
\begin{aligned}
& \left(X_{R} \otimes Y_{K}\right) \otimes Z_{L}=X_{R} \otimes\left(Y_{K} \otimes Z_{L}\right) \\
& \left(X_{R} \oplus Y_{R}\right) \otimes Z_{L}=X_{R} \otimes Z_{L} \oplus Y_{R} \otimes Z_{L} \\
& Z_{L} \otimes\left(X_{R} \oplus Y_{R}\right)=Z_{L} \otimes X_{R} \oplus Z_{L} \otimes Y_{R}
\end{aligned}
$$

We point out that the chain complex $k=(0 \rightarrow k)$ is a unit for the tensor product, that is

$$
X_{R} \otimes k=X_{R}=k \otimes X_{R}
$$

Here we use the obvious identification $V \otimes k=V=k \otimes V$ for a $k$-vector space $V$.

We shall use the tensor product of secondary modules mainly for the next result.

Proposition 4.6. The cup product map $\mu: Z^{n} \times Z^{m} \rightarrow Z^{n+m}$ induces an $i_{n, m}{ }^{-}$ equivariant map between secondary modules

$$
\mu_{*}: \mathcal{H}^{n}(X) \otimes \mathcal{H}^{m}(X) \rightarrow \mathcal{H}^{n+m}(X)
$$

where $i_{n, m}: k\left[\sigma_{n}\right] \otimes k\left[\sigma_{m}\right] \rightarrow k\left[\sigma_{n+m}\right]$ is induced by the inclusion $\sigma_{n} \times \sigma_{m} \subset \sigma_{n+m}$, $n \geqslant 1$.

Proof. The map $\mu_{*}$ carries $f \otimes g \in \mathcal{H}_{0}^{n} \otimes \mathcal{H}_{0}^{m}$ with $f: X \rightarrow Z^{n}, g: X \rightarrow Z^{m}$ to the composite $\mu(f, g): X \rightarrow Z^{n} \times Z^{m} \rightarrow Z^{n+m}$. Since $\mu$ is $k$-bilinear and $i_{n, m^{-}}$ equivariant $\mu_{*}: \mathcal{H}_{0}^{n} \otimes \mathcal{H}_{0}^{m} \rightarrow \mathcal{H}_{0}^{n+m}$ is well defined. Moreover $\mu_{*}$ is induced on $\left(\mathcal{H}^{n} \otimes \mathcal{H}^{m}\right)_{1}$ by the map

$$
\bar{\mu}: \mathcal{H}_{0}^{n} \otimes \mathcal{H}_{1}^{m} \oplus \mathcal{H}_{1}^{n} \otimes \mathcal{H}_{0}^{m} \rightarrow \mathcal{H}_{1}^{n+m}
$$

which carries $f \otimes G$ with $G: g \Rightarrow 0 \in \mathcal{H}_{1}^{m}$ to $\mu(f, G): \mu(f, g) \Rightarrow 0$ and carries $H \otimes g$ with $H: f \Rightarrow 0 \in \mathcal{H}_{1}^{n}$ to $\mu(H, g): \mu(f, g) \Rightarrow 0$. Here we use the fact that the bilinearity of $\mu$ implies that $\mu(f, 0)=0=\mu(0, g)$. If $H: f \Rightarrow 0$ and $G: g \Rightarrow 0$ are given then in fact

$$
\bar{\mu}(f \otimes G)=\bar{\mu}(H \otimes g)
$$


so that $\mu_{*}$ is well defined and $i_{n, m}$-equivariant. In fact, we have for the track $(H, G)$ : $(f, g) \Rightarrow(0,0)$ with $(f, g): X \rightarrow Z^{n} \times Z^{m}$ given by the homotopy $\left(H_{t}, G_{t}\right)$ the formula

$$
\begin{aligned}
\mu(H, G) & =\mu((0, G) \square(H, g)) \\
& =\mu((H, 0) \square(f, G))
\end{aligned}
$$

where $\square$ denotes addition of tracks. Hence we get

$$
\begin{aligned}
\mu(H, g) & =0 \square \mu(H, g)=\mu((0, G)) \square \mu(H, g) \\
& =\mu((0, G) \square(H, g))=\mu(H, G) \\
& =\mu((H, 0) \square(f, G)) \\
& =\mu(H, 0) \square \mu(f, G) \\
& =0 \square \mu(f, G)=\mu(f, G)
\end{aligned}
$$

and this proves (2). Finally we have to show that $\mu_{*}$ is compatible with the $\Gamma-$ operator. For this let $r \in R_{n}$ and $s \in R_{m}$ and let

$$
\begin{gathered}
\Gamma_{r-\varepsilon r}: r-\varepsilon r \Rightarrow 0: Z^{n} \rightarrow Z^{n} \\
\Gamma_{s-\varepsilon s}: s-\varepsilon s \Rightarrow 0: Z^{m} \rightarrow Z^{m} \\
\Gamma_{r \odot s-\varepsilon(r) \cdot \varepsilon(s)}: r \odot s-\varepsilon(r \odot s) \Rightarrow 0: Z^{n+m} \rightarrow Z^{n+m}
\end{gathered}
$$

where $r \odot s=i_{n, m}(r, s) \in R_{n+m}$. We observe that in $R_{n+m}$ we have the following equations

$$
\begin{aligned}
(r-\varepsilon r) \odot s+\varepsilon(r)\left(1_{n} \odot(s-\varepsilon s)\right) & = \\
(r \odot s) & -\varepsilon(r)\left(1_{n} \odot s\right)+\varepsilon(r)\left(1_{n} \odot s\right)-\varepsilon(r) \varepsilon(s)\left(1_{n} \odot 1_{m}\right) \\
& =r \odot s-\varepsilon(r \odot s) \in R_{n+m}
\end{aligned}
$$

Let $Z^{n} \wedge Z^{m}=Z^{n} \times Z^{m} / Z^{n} \times\{0\} \cup\{0\} \times Z^{m}$ be the smash product. Then the cup product map $\mu$ induces a map $\widetilde{\mu}: Z^{n} \wedge Z^{m} \rightarrow Z^{n+m}$ and we get the composites $a, b, c: Z^{n} \wedge Z^{m} \rightarrow Z^{n+m}$ by

$$
\begin{aligned}
a & =\widetilde{\mu}(r-\varepsilon r) \wedge s, \\
b & =\varepsilon(r) \widetilde{\mu}\left(1_{n} \wedge(s-\varepsilon s)\right), \\
c & =(r \odot s-\varepsilon(r \odot s)) \widetilde{\mu} .
\end{aligned}
$$

Then (5) shows that $a+b=c$. Hence

$$
\begin{aligned}
& A=\widetilde{\mu}\left(\Gamma_{r-\varepsilon r} \wedge s\right)+\varepsilon(r) \widetilde{\mu}\left(1_{n} \wedge \Gamma_{s-\varepsilon s}\right) \text { and } \\
& B=\Gamma_{r \odot s-\varepsilon(r \odot s)} \widetilde{\mu}
\end{aligned}
$$

are both tracks $c \Rightarrow 0$. Now obstruction theory shows that these tracks $A$ and $B$ coincide since the set of homotopy classes $\left[\Sigma Z^{n} \wedge Z^{m}, Z^{n+m}\right]$ is trivial. The equation $A=B$ implies that $\mu$ satisfies

$$
\mu_{*} \Gamma(r \otimes s \otimes f \otimes g)=\Gamma(r \odot s \otimes \mu(f, g))
$$

by definition on $\Gamma$ in (2.6) and by formula (2.4), which exactly corresponds to $A=B$. 


\section{Secondary cohomology}

Using the tensor product of secondary modules we introduce the notion of a secondary algebra. We define a functor which associates with each space $X$ a secondary cohomology algebra $\mathcal{H}^{*}(X)$.

We consider a sequence $R_{*}$ of augmented $k$-algebras $R_{n}, n \geqslant 0$, together with augmented algebra maps

$$
i_{n, m}=\odot: R_{n} \otimes R_{m} \rightarrow R_{n+m}
$$

carrying $\alpha \otimes \beta$ to $\alpha \odot \beta$ such that for $\gamma \in R_{k}$ we have

$$
(\alpha \odot \beta) \odot \gamma=\alpha \odot(\beta \odot \gamma)
$$

in $R_{n+m+k}$. Since $\odot$ is an algebra map we have

$$
\left(\alpha \cdot \alpha^{\prime}\right) \odot\left(\beta \cdot \beta^{\prime}\right)=(\alpha \odot \beta) \cdot\left(\alpha^{\prime} \odot \beta^{\prime}\right)
$$

where $\alpha \cdot \alpha^{\prime}$ denotes the product in $R_{n}$. Let $1_{n} \in R_{n}$ be the unit element of $R_{n}$ with $1_{n} \odot 1_{m}=1_{n+m}$. For $n=0$ we have $R_{0}=k$ and $1_{0} \in R_{0}$ satisfies $1_{0} \odot \alpha=\alpha \odot 1_{0}=\alpha$. We call $R_{*}=\left(R_{*}, \odot\right)$ a coefficient algebra.

Of course we have the trivial coefficient algebra $k$ with $R_{n}=k$ for $n \geqslant 0$. On the other hand we shall use the symmetric coefficient algebra $k\left[\sigma_{*}\right]$ given by the augmented group algebras $R_{n}=k\left[\sigma_{n}\right]$ where $\sigma_{n}$ is the symmetric group and $R_{n}$ has the sign augmentation (1.1)(3). Moreover $\odot=i_{m, n}$ is induced by the inclusion of groups $\sigma_{n} \times \sigma_{m} \subset \sigma_{n+m}$.

Definition 5.2. An algebra $V$ over a coefficient algebra $R_{*}$ is a sequence of $R_{n^{-}}$ modules $V^{n}, n \geqslant 0$, together with $k$-linear maps

$$
V^{n} \otimes V^{m} \rightarrow V^{n+m}
$$

carrying $x \otimes y$ to $x \cdot y$. For $z \in V^{k}$ we have in $V^{n+m+k}$

$$
(x \cdot y) \cdot z=x \cdot(y \cdot z)
$$

and for $\alpha \in R_{n}, \beta \in R_{m}$ we have

$$
(\alpha x) \cdot(\beta y)=(\alpha \odot \beta)(x \cdot y) .
$$

We do not assume that the algebra $V$ has a unit. Let $V$ and $W$ be such algebras over $R_{*}$. Then a map $f: V \rightarrow W$ over $R_{*}$ is given by an $R_{n}$-linear map $f=f^{n}$ : $V^{n} \rightarrow W^{n}, n \geqslant 0$, with $f(x \cdot y)=f(x) \cdot f(y)$. This defines the category of algebras over $R_{*}$.

If $R_{*}=k$ is the trivial coefficient algebras then $V$ in (5.2) is just a graded algebra over $k$. A graded algebra $V$ is commutative if for $x \in V^{n}, y \in V^{m}$ we have

$$
y \cdot x=(-1)^{n m} x \cdot y
$$

For example the reduced cohomology $\widetilde{H}^{*}(X, k)$ of a pointed space $X$ with coefficients in $k$ is a commutative graded algebra. We generalize this notion of commutative algebras as follows. 
Definition 5.4. Let $R_{*}$ be a coefficient algebra and assume elements

$$
\tau_{m, n} \in R_{n+m}(n, m \geqslant 0)
$$

are given with the following properties $(m, n, k \geqslant 0)$.

$$
\begin{aligned}
\tau_{m, n} \tau_{n, m} & =1_{n+m} \\
\tau_{m, 0}=\tau_{0, m} & =1_{m} \\
\tau_{n, m}(\alpha \odot \beta) & =(\beta \odot \alpha) \tau_{n, m} \text { for } \alpha \in R_{n}, \beta \in R_{m} \\
\tau_{m+n, k} & =\left(\tau_{m, k} \odot 1_{n}\right)\left(1_{m} \odot \tau_{n, k}\right) \\
\varepsilon\left(\tau_{m, n}\right) & =(-1)^{m, n} \in k
\end{aligned}
$$

Then we say that an algebra $V$ over $R_{*}$ is $\tau$-commutative if for $x \in V^{n}, y \in V^{m}$

$$
y \cdot x=\tau_{n, m}(x \cdot y)
$$

in $V^{n+m}$.

For example we have the interchange elements $\tau_{n, m} \in k\left[\sigma_{n+m}\right]$ with $\tau_{n, m}(1)=$ $m+1$ in the symmetric coefficient algebra for which a $\tau$-commutative algebra is the same as a "commutative twisted algebra" in the sense of Stover [St]. On the other hand we can define the interchange elements $\tau_{n, m}=(-1)^{n m} \in k$ in the trivial coefficient algebra so that in this case a $\tau$-commutative algebra is the same as a commutative graded algebra in (5.3). We now are ready to introduce the notion of a secondary algebra.

Definition 5.5. Let $R_{*}$ be a coefficient algebra. A secondary algebra $\mathcal{H}^{*}$ over $R_{*}$ consists of a sequence of secondary modules $\mathcal{H}^{n}$ over $R_{n}, n \geqslant 1$, together with $i_{n, m}$-equivariant maps

$$
\mu=\mu_{n, m}: \mathcal{H}^{n} \otimes \mathcal{H}^{m} \rightarrow \mathcal{H}^{n+m}
$$

for $n, m \geqslant 1$ which are associative in the sense that the diagram

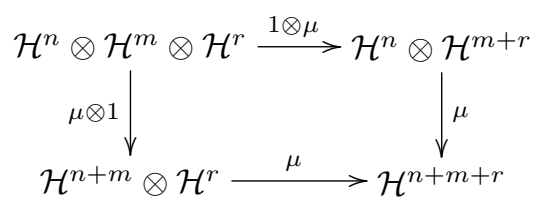

commutes. Here we use the tensor product of secondary modules. If elements $\tau_{n, m} \in$ $R_{n+m}$ are given as in (5.4) we say that the secondary algebra $\mathcal{H}^{*}$ is $\tau$-commutative if the diagram

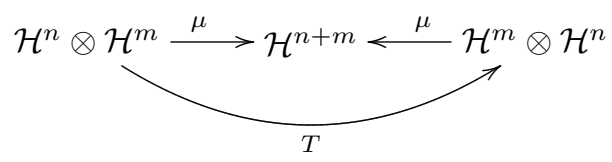

commutes. Here $T$ carries $x \otimes y$ to $\tau_{n, m}(y \otimes x)$ for $\left(x \in \mathcal{H}_{0}^{n}, y \in \mathcal{H}_{0}^{m}\right)$ or $\left(x \in \mathcal{H}_{0}^{n}, y \in\right.$ $\left.\mathcal{H}_{1}^{m}\right)$ or $\left(x \in \mathcal{H}_{1}^{n}, y \in \mathcal{H}_{0}^{m}\right)$. We study $\tau$-commutative secondary algebras in more detail in section $\S 7$ below. 
We say that the secondary algebra $\mathcal{H}^{*}$ is $w$-closed if one has $k$-linear isomorphisms

$$
w=w^{n}: \pi_{1} \mathcal{H}^{n} \cong \pi_{0} \mathcal{H}^{n-1}
$$

for $n \geqslant 1$ which satisfy

$$
\begin{aligned}
& w^{n+m}\left(y_{1} \cdot z\right)=w^{n}\left(y_{1}\right) \cdot z \\
& w^{n+m}\left(y \cdot z_{1}\right)=(-1)^{n} y \cdot w^{m}\left(z_{1}\right)
\end{aligned}
$$

for $y \in \pi_{0} \mathcal{H}^{n}, z \in \pi_{0} \mathcal{H}^{m}, y_{1} \in \pi_{1} \mathcal{H}^{n}, z_{1} \in \pi_{1} \mathcal{H}^{m}$. In (5) the multiplication is defined by the maps (1). A map $f: \mathcal{H}^{*} \rightarrow \mathcal{G}^{*}$ between secondary algebras is given by a sequence $f^{n}: \mathcal{H}^{n} \rightarrow \mathcal{G}^{n}$ of $R_{n}$-equivariant maps between secondary modules such that $f^{n}$ is compatible with $\mu$ in (1) and $w$ in (4). Such a map $f$ is a weak equivalence if $f^{n}$ is a weak equivalence in secmod for $n \geqslant 0$.

Let secalg be the category of secondary algebras over the symmetric coefficient algebra $k\left[\sigma_{*}\right]$ which are $\tau$-commutative and $w$-closed. For an object $\mathcal{H}^{*}$ in secalg we obtain a commutative graded algebra $H^{*}$ by

$$
H^{n}=\pi_{0} \mathcal{H}^{n} \text { for } n \geqslant 0
$$

with the multiplication $H^{n} \otimes H^{m} \rightarrow H^{n+m}$ induced by $\mu$ in (5.5)(1). We see that $H^{*}$ is commutative since $\mathcal{H}^{*}$ is $\tau$-commutative. Assume $\operatorname{sign}\left(\tau_{n, m}\right)=1$ in $k$ then $\mu: \mathcal{H}^{n} \otimes \mathcal{H}^{n} \rightarrow \mathcal{H}^{2 n}$ for $n \geqslant 1$ yields the squaring operation

$$
\mathrm{Sq}^{n-1}=w^{2 n-1} \mathrm{Sq}: H^{n} \rightarrow H^{2 n-1}
$$

with $2 \mathrm{Sq}^{n-1}=0$ as follows. For this we use the assumption that $\mathcal{H}^{*}$ is $w$-closed. The $k$-linear map

$$
S q: \pi_{0} \mathcal{H}^{n} \longrightarrow \pi_{1} \mathcal{H}^{2 n}
$$

carries the element $\{y\}$ represented by $y \in \mathcal{H}_{0}^{n}$ to the element

$$
S q\{y\}=\Gamma\left(\tau_{n, n}, y \cdot y\right)
$$

wich satisfies $\partial \Gamma\left(\tau_{n, n}, y \cdot y\right)=0$. One can check that $S q$ is well defined, see also [B]. The next result describes the secondary cohomology algebra $\mathcal{H}^{*}(X)$ of a pathconnected pointed space $X$. Let Top $_{0}^{*}$ be the category of path-connected pointed spaces and pointed maps.

Theorem 5.8. There is a contravariant functor

$$
\mathcal{H}^{*}: \operatorname{Top}_{0}^{*} \rightarrow \text { secalg }
$$

which carries a space $X$ to a secondary algebra $\mathcal{H}^{*}(X)$ which is $\tau$-commutative and $w$-closed. See (4.6). Moreover the algebra $\widetilde{H}^{*}=\pi_{0} \mathcal{H}^{*}(X)$ is (5.6) coincides with the reduced cohomology algebra $\widetilde{H}^{*}(X ; k)$ and for $k=\mathbb{F}_{2}$ the squaring operation $\mathrm{Sq}^{n-1}$ in (5.7) coincides with the corresponding Steenrod operation. 
Proof. This is a consequence of (3.7) and property (c) of the cup product maps in the introduction. The result on Steenrod squares is a consequence of a result of Kristensen, lemma 2.5 in $[\mathbf{K}]$. Compare $[\mathbf{B}]$.

\section{Crossed modules and Hochschild cohomology}

we show that Hochschild cohomology can be deduced from the concept of secondary algebra in (5.5). More precisely, a secondary algebra over $R_{*}=k$ corresponds to the notion of a "crossed module" which is used to define "crossed extensions". Moreover weak equivalence classes of crossed extensions are in fact the elements in the Hochschild cohomology. In a similar way we shall deduce from the concept of a $\tau$-commutative secondary algebra in (5.5) the notion of symmetric cohomology; see $\S 9$ below.

We introduce the concept of a crossed module in the context of algebras and we show that (in the graded case) a crossed module is the same as a secondary algebra over the trivial coefficient algebra $R_{*}=k$. A crossed module and equivalently a secondary algebra over $k$ represent an element in the third Hochschild cohomology. This leads to the notion of a characteristic class of a differential algebra.

We here consider the graded and the non-graded case at the same time. A graded vector space $V$ is assumed to be non-negatively graded, i. e. $V^{i}=0$ for $i<0$.

We use the following notation. An algebra $\widetilde{A}$ is given by a (graded) $k$-vector space $\widetilde{A}$ and a multiplication map $\widetilde{A} \otimes \widetilde{A} \rightarrow \widetilde{A}$ which is associative. On the other hand a $k$-algebra $A$ is an algebra with unit $k \rightarrow A$ and augmentation $\varepsilon: A \rightarrow k$. Hence a $k$-algebra $A$ is an algebra under and over $k$. Then the augmentation ideal

$$
\widetilde{A}=\operatorname{kernel}(\varepsilon: A \rightarrow k)
$$

is an algebra which determines the $k$-algebra $A=\widetilde{A} \oplus k$ completely. Moreover an $\widetilde{A}$-module is also an $A$-module and vice versa.

Let $A$ be a (graded) $k$-algebra. An $A$-bimodule $V$ is a (graded) $k$-vector space which is a left and a right $A$-module such that for $a, b \in A, x \in V$ we have $(a \cdot x) \cdot b=$ $a \cdot(x \cdot b)$. For example $A$ can be considered as an $A$-bimodule via the multiplication in $A$.

Definition 6.1. A crossed module is a map of $A$-bimodules

$$
\partial: V \rightarrow A
$$

satisfying $\varepsilon \partial=0$ and $(\partial v) \cdot w=v \cdot(\partial w)$ for $v, w \in V$.

Let $\pi_{0}(\partial)=\operatorname{cokernel}(\partial)$ and $\pi_{1}(\partial)=\operatorname{kernel}(\partial)$ in the category of (graded) vector spaces. Then the algebra structure of $A$ induces an algebra structure of $\pi_{0}(\partial)$ and the $A$-bimodule structure of $V$ induces a $\pi_{0}(\partial)$-bimodule structure of $\pi_{1}(\partial)$. In fact for $\{a\} \in \pi_{0}(\partial)$ the multiplication $\{a\} \cdot v=a \cdot v$ with $v \in \pi_{1}(\partial)$ is well defined since $(a+\partial w) \cdot v=a \cdot v+(\partial w) \cdot v=a \cdot v+w \cdot \partial v=a \cdot v$ where $\partial v=0$. Hence a crossed module yields the exact sequence

$$
0 \longrightarrow \pi_{1}(\partial) \stackrel{i}{\longrightarrow} V \stackrel{\partial}{\longrightarrow} A \stackrel{q}{\longrightarrow} \pi_{0}(\partial) \longrightarrow 0
$$


in which all maps are $A$-bimodule morphisms. Here the $A$-bimodule structure of $\pi_{0}(\partial)$ and $\pi_{1}(\partial)$ is induced by the algebra map $q$.

Lemma 6.2. A secondary algebra $\mathcal{H}^{*}$ over the trivial coefficient algebra $k$ as defined in (5.5) is the same as a crossed module $\partial$.

Proof. Given $\mathcal{H}^{*}$ we obtain

$$
\partial: \mathcal{H}_{1}^{*} \rightarrow \mathcal{H}_{0}^{*}
$$

where $\mathcal{H}_{0}^{*}$ is an algebra by the multiplication $\mu$ in $(5.5)(1)$. Moreover $\mathcal{H}_{1}^{*}$ is a $\mathcal{H}_{0}^{*-}$ bimodule by the multiplication (5.5)(1). Using (4.1) we see that $\partial$ yields the crossed module

$$
\partial=(\partial, 0): \mathcal{H}_{1}^{*} \rightarrow \mathcal{H}_{0}^{*} \oplus k
$$

where $\mathcal{H}_{0}^{*} \oplus k$ is the $k$-algebra given by $\mathcal{H}_{0}^{*}$. Conversely it is easy to see that a crossed module (6.1) defines a secondary algebra over $k$.

We now use crossed modules (or equivalently secondary algebras over $k$ ) for the definition of Hochschild cohomology.

Definition 6.3. Let $H$ be a (graded) $k$-algebra and let $M$ be an $H$-bimodule. A crossed extension of $H$ by $M$ is an exact sequence in the category of (graded) $k$-vector spaces

$$
\mathcal{E}: 0 \longrightarrow M \stackrel{\gamma}{\longrightarrow} V \stackrel{\partial}{\longrightarrow} A \stackrel{q}{\longrightarrow} H \longrightarrow 0
$$

where $\partial$ is a crossed module. Moreover all maps are $A$-bimodule maps with the $A$-bimodule structure induced by the algebra map $q: A \rightarrow H$. A weak equivalence between two such extensions is a commutative diagram

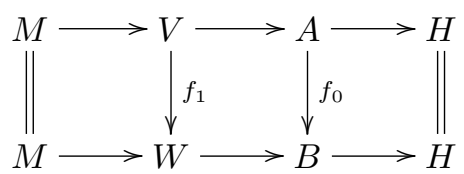

where $f_{0}$ is an algebra map and $f_{1}$ is a $f_{0}$-biequivariant homomorphism.

induces an isomorphism $\operatorname{kernel}\left(q^{\prime}\right)=\operatorname{kernel}(q)$ and hence we get for an $n$-fold extension (6.3) the following diagram of $G$ by $f^{*} M$. Now $f^{*}$ in (6.4) carries the weak-equivalence class of the extension $\mathcal{E}$ to the weak-equivalence class of the extension $f^{*} \mathcal{E}$ in the top row of the diagram. $(n \geqslant 2)$ weak equivalence class of the extension $\mathcal{E}$ to the weak equivalence class of the extension $g_{*} \mathcal{E}$ in the bottom row of the diagram.

$M, n \geqslant 2$.

Proposition 6.4. The third Hochschild cohomology $H H^{3}(H, M)$ of $H$ with coefficients in $M$ coincides naturally with the set of weak equivalence classes of crossed extensions of $H$ by $M$.

This result is proved in $[\mathbf{B M}]$, see also Loday $[\mathbf{L}]$ or Lue $[\mathbf{L u}]$. The proposition holds in the graded and in the non-graded case.in order to define a crossed resolution 
of a $k$-algebra $A$. tensor product of $V$. Given a (graded) $k$-algebra $A$ and a $k-$ linear map $d: V \rightarrow A$ with $\varepsilon d=0$ we obtain the free crossed modul with basis $(V, d)$ as follows. Let is the free crossed module with basis $(V, d)$. Finally we define for a $k$-algebra $H$ the free $H$-bimodule with basis $V$ by $H \otimes V \otimes H$. choose a commutative diagram $(n \geqslant 2)$ this surjection we define the $k$-vector space structure of $\mathrm{HH}^{n+1}(H, M)$ so that addition of crossed extensions in $\mathrm{HH}^{n+1}(H, M)$ is given by the "Baer sum" of extensions. 0

$h_{0}(v)=1 \otimes v \otimes 1$ for $v \in V_{0}$ and $h_{0}(a \cdot b)=(q a) \cdot h_{0}(b)+h_{0}(b) \cdot q(a)$ for $a, b \in T$. One can check that there is a unique $H$-bimodule map $d_{2}$ for which

We have seen in (6.2) that each secondary algebra $\mathcal{H}^{*}$ over $k$ yields a canonical crossed extension

$$
0 \longrightarrow \pi_{1}\left(\mathcal{H}^{*}\right) \longrightarrow \mathcal{H}_{1}^{*} \stackrel{\partial}{\longrightarrow} \mathcal{H}_{0}^{*} \oplus k \longrightarrow \pi_{0}\left(\mathcal{H}^{*}\right) \oplus k \longrightarrow 0
$$

Here $H=\pi_{0}\left(\mathcal{H}^{*}\right) \oplus k$ is a $k$-algebra and $M=\pi_{1}\left(\mathcal{H}^{*}\right)$ is an $H$-bimodule. Hence the crossed extension represents an element

$$
\left\langle\mathcal{H}^{*}\right\rangle \in \mathrm{HH}^{3}(H, M)
$$

which is termed the characteristic class of the secondary algebra $\mathcal{H}^{*}$. On the other hand a differential algebra $C$ (like the cochain algebra of a space) as well yields a crossed extension representing a characteristic class $\langle C\rangle$ as in the following example.

Example 6.6. Let $C$ be a differential graded $k$-algebra, that is, $C=\left\{C^{i}, i \geqslant 0\right\}$ with $C^{i} C^{j} \subseteq C^{i+j}$ and $d: C \rightarrow C$ of degree +1 satisfying $d(x y)=(d x) y+$ $(-1)^{|x|} x d(y)$ and $d d=0$ and $\varepsilon d=0$. Then $d$ induces the map of graded $k$-vector spaces

$$
V=\operatorname{coker}(\widetilde{d})[1] \stackrel{\partial}{\longrightarrow} \operatorname{ker}(d)=A
$$

Here we define for a graded vector space $W$ the shifted graded vector space $W[1]$ by

$$
W^{n}=(W[1])^{n+1}, w \mapsto s(w),
$$

Hence for the cokernel of the differential $\operatorname{coker}(d)=\widetilde{C} / \mathrm{im}(\widetilde{d})$ we obtain the shifted object $V=\operatorname{coker}(\widetilde{d})[1]$. Since $d$ is of degree +1 the boundary induces $\partial$ by $\partial s\{v\}=$ $d(v)$ for $\{v\} \in \operatorname{coker}(d), v \in C$. The algebra $C$ induces an algebra structure of $A=\operatorname{ker}(d)$. Moreover it induces the structure of an $A$-bimodule on $V$ by setting

$$
\begin{aligned}
a \cdot(s\{v\}) & =(-1)^{|a|} s\{a \cdot v\} \\
(s\{v\}) \cdot b & =s\{v \cdot b\}
\end{aligned}
$$

One can check that $\partial: V \rightarrow A$ is a crossed module in the sense of (6.1), see [BM]. This proves that $\partial: V \rightarrow A$ is crossed module and therefore we obtain by (6.2) a secondary algebra $\partial: V \rightarrow \widetilde{A}$ over $k$ which is, in fact, $w$-closed (see (5.5)) by defining

$$
w: \pi_{1}(\partial)=\widetilde{H}^{*}(C)[1] \cong \widetilde{H}^{*}(C)=\pi_{0}(\partial)
$$

with $w s(x)=x$ for $x \in H^{*}(C)=\operatorname{ker}(d) / \operatorname{im}(d)$. The equations in (3) for the $A$-bimodule structure of $V$ correspond exactly to the equations in (5.5)(5). 
According to (3) we define for a graded algebra $H^{*}$ the $H^{*}$-bimodule $\widetilde{H}^{*}[1]$ by setting

$$
\begin{aligned}
a \cdot(s x) & =(-1)^{|a|} s(a \cdot x) \\
(s x) \cdot b & =s(x \cdot b)
\end{aligned}
$$

Then we obtain by (1) and (4) the crossed 2-extension

$$
0 \longrightarrow \widetilde{H}^{*}[1] \longrightarrow V \stackrel{\partial}{\longrightarrow} A \longrightarrow H^{*} \longrightarrow 0
$$

which by (6.4) represents an element

$$
\langle C\rangle \in \mathrm{HH}^{*}\left(H^{*}, \widetilde{H}^{*}[1]\right)
$$

where $H^{*}=H^{*} C$ is the cohomology algebra of the differential algebra $C$. A cocycle $\theta$ representing $\langle C\rangle$ is considered in Berrick-Davydov [BD].

As a special case we obtain for a pointed space $X$ the augmented algebra of cochains on $X$ denoted by $C^{*} X$ for which

$$
H^{*}\left(C^{*} X\right)=H^{*}(X)
$$

is the cohomology algebra of $X$. Hence we get by (6.6)(6) the class

$$
\left\langle C^{*}(X)\right\rangle \in \mathrm{HH}^{*}\left(H^{*}(X), \widetilde{H}^{*}(X)[1]\right)
$$

which is an invariant of the homotopy type of $X$ in the sense that a pointed map $f: X \rightarrow Y$ satisfies

$$
\left(f^{*}\right)^{*}\left\langle C^{*}(X)\right\rangle=\left(f^{*}[1]\right) *\left\langle C^{*}(Y)\right\rangle
$$

in $\mathrm{HH}^{3}\left(H^{*}(Y), \widetilde{H}^{*}(X)[1]\right)$ where $f^{*}: H^{*}(Y) \rightarrow H^{*}(X)$ yields the structure of an $H^{*}(Y)$-bimodule on $\widetilde{H}^{*}(X)[1]$.

We now compare the class (6.7) with the secondary cohomology algebra $\mathcal{H}^{*}(X)$ in (5.8).

Proposition 6.8. By forgetting structure we obtain from the secondary cohomology $\mathcal{H}^{*}(X)$ a secondary algebra over $k$ denoted by $\mathcal{H}^{*}(X)_{(k)}$. Then the classes

$$
\left\langle C^{*} X\right\rangle=\left\langle\mathcal{H}^{*}(X)_{(k)}\right\rangle \in \mathrm{HH}^{3}\left(H^{*}(X), \widetilde{H}^{*}(X)[1]\right)
$$

given by (6.5) and (6.7) coincide.

Proof. Using (3.3) and the definition of $Y_{3}$ in Baues [B] we see that $\left\langle\mathcal{H}\left(X, Y_{3}\right)\right\rangle=$ $\left\langle C^{*} X\right\rangle$ for a simplicial set $X$. Here we use the universal property of $Y_{3}$ which says that a simplicial map $X \rightarrow Y_{3}$ can be identified with a cocycle in $C^{*} X$.

\section{7. $\tau$-crossed modules for commutative graded algebras}

directly be obtained by crossed $n$-fold extensions of $H$ by $M$. A crossed module which by (6.2) is the same as a secondary algebra over $k$ was the crucial ingredient of a crossed extension. We now simply replace the "secondary algebra over $k$ " in a crossed extension by a " $\tau$-commutative secondary algebra" and we then 
obtain $\tau$-crossed extensions which represent elements in the symmetric cohomology $\mathrm{SH}^{*}(H, M)$.

We have seen in $\S 7$ that a secondary algebra over the trivial coefficient algebra $k$ is the same as a crossed module. We here show that in a similar way a $\tau$-commutative secondary algebra over $R_{*}$ is the same as a $\tau$-crossed module. Weak equivalence classes of $\tau$-crossed modules yield an abelian group generalizing the Hochschild cohomology in (6.4).

Let $R_{*}$ be a coefficient algebra with interchange elements $\tau_{m, n} \in R_{m+n}$, for example let $R_{*}=k\left[\sigma_{*}\right]$ be the symmetric coefficient algebra. An $R_{*}-$ module $V$ is a sequence of (left) $R_{n}$-modules $V^{n}, n \geqslant 0$. A map or an $R_{*}$-linear map $f: V \rightarrow W$ between $R_{*}$-modules is given by a sequence of $R_{n}$-linear maps $f^{n}: V^{n} \rightarrow W^{n}$ for $n \geqslant 0$. The field $k$ (concentrated in degree 0 ) is an $R_{*}$-module. Moreover using the augmentation $\varepsilon$ of $R_{n}, n \geqslant 0$, we see that each graded $k$-vector space $M$ is an $R_{*}-$ module which we call an $\varepsilon-$ module. For $x \in M^{m}$ we write $|x|=m$ where $|x|$ is the degree of $x$.

Given $R_{*}$-modules $V_{1}, \ldots, V_{k}$ we define the $R_{*}$-tensor product $V_{1} \bar{\otimes} \ldots \bar{\otimes} V_{k}$ by

$$
\left(V_{1} \bar{\otimes} \ldots \bar{\otimes} V_{k}\right)_{n}=\bigoplus_{n_{1}+\ldots+n_{k}=n} R_{n} \otimes_{R_{n_{1}} \otimes \ldots \otimes R_{n_{k}}} V_{1}^{n_{1}} \otimes \ldots \otimes V_{k}^{n_{k}}
$$

where we use the algebra map $\odot: R_{n_{1}} \otimes \ldots \otimes R_{n_{k}} \rightarrow R_{n}$ given by the structure of the coefficient algebra $R_{*}$ in (5.1). One readily checks associativity

$$
\left(V_{1,1} \bar{\otimes} \ldots \bar{\otimes} V_{1, k_{1}}\right) \bar{\otimes} \ldots \bar{\otimes}\left(V_{s, 1} \bar{\otimes} \ldots \bar{\otimes} V_{s, k_{s}}\right)=V_{1,1} \bar{\otimes} \ldots \bar{\otimes} V_{1, k_{1}} \bar{\otimes} \ldots \bar{\otimes} V_{s, 1} \bar{\otimes} \ldots \bar{\otimes} V_{s, k_{s}}
$$

Compare Stover $[\mathbf{S t}]$ 2.9. Moreover the interchange element $\tau$ in $R_{*}$ yields the isomorphism

$$
T: V \bar{\otimes} W \cong W \bar{\otimes} V
$$

which carries $v \otimes w$ to $\tau_{w, v} w \otimes v$ where

$$
\tau_{w, v}=\tau_{m, n} \in R_{m+n}
$$

for $w \in W^{m}, v \in V^{n}$. Of course we have $k \bar{\otimes} V=V=V \bar{\otimes} k$.

Definition 7.2. An algebra $A$ over $R_{*}$ is given by an $R_{*}$-linear map $\mu: A \bar{\otimes} A \rightarrow$ $A, \mu(a \otimes b)=a \cdot b$, which is associative in the sense that the diagram

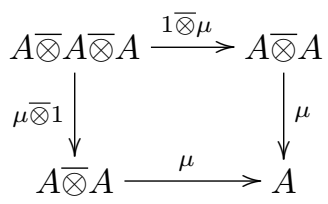

commutes. Moreover $A$ is $\tau-$ commutative if

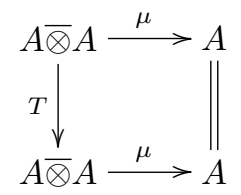


commutes. One readily checks that this coincides with the notation in (5.2) and (5.3). We say that $A$ is a $k$-algebra over $R_{*}$ if algebra maps $k \stackrel{i}{\longrightarrow} A \stackrel{\varepsilon}{\longrightarrow} k$ are given with $\varepsilon i=1$. Such a $k$-algebra $A$ over $R_{*}$ is completely determined by the algebra $\widetilde{A}$ over $R_{*}$ with $\widetilde{A}=\operatorname{kernel}(\varepsilon: A \rightarrow k)$ and $A=k \oplus \widetilde{A}$.

For an $R_{*}$-module $V$ let $V_{(k)}$ be the underlying graded $k$-vectorspace. If $A$ is a $k$-algebra over $R_{*}$ then $A_{(k)}$ is a $k$-algebra (over $k$ ) in the sense of $\S 7$ above.

Definition 7.3. Given an algebra $A$ over $R_{*}$ we say that an $R_{*}-$ module $V$ is an $A-$ module if a map $m: A \bar{\otimes} V \rightarrow V$ is given such that

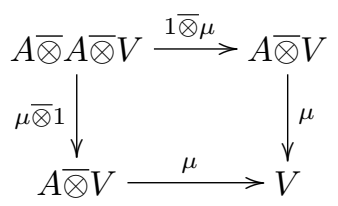

commutes. Hence for $a \cdot x=\mu(a \otimes x)$ with $a \in A, x \in V$ we have $(\alpha a) \cdot(\beta x)=$ $(\alpha \odot \beta)(a \cdot x)$ and $(a \cdot b) \cdot x=a \cdot(b \cdot x)$. If $A$ is a $k$-algebra over $R_{*}$ we also assume that $1 \cdot x=x$ for $1 \in k, x \in V$. Then the $A$-module $V$ is an $\widetilde{A}$-module and vice versa. In particular the algebra $A$ is also an $A$-module in the obvious way.

Lemma 7.4. Let $A$ be a $\tau$-commutative $k$-algebra over $R_{*}$ and let $V$ be an $A$ module. Then $V_{(k)}$ is a $A_{(k)}$-bimodule by defining

$$
a \cdot x \cdot b=a \cdot \tau_{b, x}(b \cdot x)
$$

for $a, b \in A, x \in V$.

Proof. We write $1_{x}=1_{n} \in R_{n}$ for $x \in V^{n}$. Now we have for $a, b \in A$

$$
\begin{aligned}
(a \cdot x) \cdot b & =\tau_{b, a \cdot x} b \cdot(a \cdot x)=\tau_{b, a \cdot x}(b \cdot a) \cdot x \\
& =\tau_{b, a \cdot x}\left(\tau_{a, b} a \cdot b\right) \cdot x \\
& =\tau_{b, a \cdot x}\left(\tau_{a, b} \odot 1_{x}\right)(a \cdot b \cdot x) \\
a \cdot(x \cdot b) & =a \cdot \tau_{b, x}(b \cdot x)=\left(1_{a} \odot \tau_{b, x}\right)(a \cdot b \cdot x)
\end{aligned}
$$

Here we have $\tau_{b, a \cdot x}\left(\tau_{a, b} \odot 1_{x}\right)=1_{a} \odot \tau_{b, x}$ by one of the equations in (6.4).

If $A$ and $V$ in (7.4) are $\varepsilon-$ modules then (7.4) corresponds to the following special case.

Lemma 7.5. Let $H$ be a commutative graded $k$-algebra and let $M$ be an $H$-module. Then $M$ is an $H$-bimodule by defining

$$
a \cdot x \cdot b=a \cdot(-1)^{|b||x|} b \cdot x
$$

for $a, b \in H$ and $x \in M$.

For an $R_{*}$-module $V$ we obtain as in (1.6) the $R_{*}$-linear map

$$
I\left(R_{*}\right) \odot_{R_{*}} V \stackrel{\mu}{\longrightarrow} V
$$

Here the left hand side is the $R_{*}$-module given in degree $n$ by $I\left(R_{n}\right) \otimes_{R_{n}} V^{n}$ ond $\mu$ carries $a \otimes x$ to $a \cdot x$. 
Definition 7.6. Let $A$ be a $\tau$-commutative $k$-algebra over $R_{*}$. A $\tau$-crossed module $\partial$ is given by a commutative diagram of $R_{*}$-linear maps

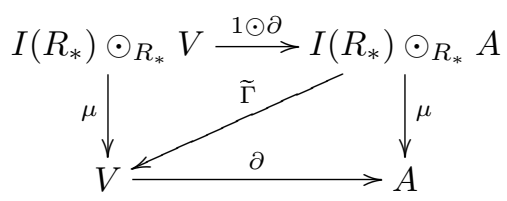

with the following properties (1) and (2). The $R_{*}-$ module $V$ is an $A$-module and $\partial$ is an $A$-module morphism, that is $\partial(a \cdot x)=a \cdot(\partial x)$ for $a \in A, x \in V$. Moreover $\varepsilon \partial=0$ and for $x, y \in V$

$$
(\partial x) \cdot y=\tau_{\partial y, x}(\partial y) \cdot x .
$$

The $R_{*}$-linear map $\widetilde{\Gamma}$ satisfies for $\beta \in I\left(R_{*}\right)$ and $a, b \in A$ the equation

$$
a \cdot \widetilde{\Gamma}(\beta \otimes b)=\widetilde{\Gamma}(1 \odot \beta \otimes a \cdot b)
$$

Equation (2) shows that $\widetilde{\Gamma}$ is a map of left $A$-modules. Moreover (1) and (2) imply that for $\alpha \in I\left(R_{*}\right)$ the following equation holds.

$$
\widetilde{\Gamma}(\alpha \otimes a) \cdot b=\widetilde{\Gamma}(\alpha \odot 1 \otimes a \cdot b)
$$

Here the right hand action of $b$ on $x=\widetilde{\Gamma}(\alpha \otimes a) \in V$ is defined as in (7.4) by $x \cdot b=\tau_{b, x} b \cdot x$. Using this notation (1) is equivalent to $(\partial x) \cdot y=x \cdot(\partial y)$, compare (6.1).

Proof of (3). For $x \in \widetilde{\Gamma}(\alpha \otimes a)$ we have $|x|=|a|$ and hence $\tau_{b, x}=\tau_{b, a}$. Therefore we get

$$
\begin{aligned}
\widetilde{\Gamma}(\alpha \otimes a) \cdot b & =\tau_{b, x} b \cdot \widetilde{\Gamma}(\alpha \otimes a) \\
& =\tau_{b, x} \widetilde{\Gamma}(1 \odot \alpha \otimes b \cdot a) \\
& =\widetilde{\Gamma}\left(\tau_{b, a}(1 \odot \alpha) \otimes b \cdot a\right) \\
& =\widetilde{\Gamma}\left((\alpha \odot 1) \tau_{b, a} \otimes b \cdot a\right) \\
& =\widetilde{\Gamma}\left((\alpha \odot 1) \otimes \tau_{b, a} b \cdot a\right) \\
& =\widetilde{\Gamma}((\alpha \odot 1 \otimes a \cdot b) .
\end{aligned}
$$

Lemma 7.8. A $\tau$-crossed module $\partial$ as in (7.6) yields for the underlying $k$-vector spaces a crossed module in the sense of (6.1)

$$
\partial: V_{(k)} \rightarrow A_{(k)}
$$

where we use (7.4). Moreover $\pi_{0}(\partial)=H$ is a commutative graded $k$-algebra and $\pi_{1}(\partial)$ is an $H$-module with the $H$-bimodule structure in (7.5).

The lemma is based on the crucial property of a $\tau$-crossed module, namely that $\pi_{0}(\partial)=\operatorname{cokernel}(\partial)$ and $\pi_{1}(\partial)=\operatorname{kernel}(\partial)$ are only $\varepsilon-$ modules though $V$ and $A$ are 
$R_{*}-$ modules. A $\tau$-crossed module yields the exact sequence

$$
0 \longrightarrow \pi_{1}(\partial) \stackrel{i}{\longrightarrow} V \stackrel{\partial}{\longrightarrow} A \stackrel{q}{\longrightarrow} \pi_{0}(\partial) \longrightarrow 0
$$

in which all maps are $A$-module morphisms. Here the $A$-module structures of $\pi_{0}(\partial)$ and $\pi_{1}(\partial)$ are induced by the algebra map $q$. Moreover for the underlying $k$-vector spaces this is by (7.8) a crossed extension as in (6.1), (6.3).

The next result generalizes lemma (6.2) on crossed modules.

Lemma 7.10. A $\tau$-commutative secondary algebra $\mathcal{H}^{*}$ over the coefficient algebra $R_{*}$ as defined in (5.5) is the same as a $\tau$-crossed module $\partial$ in (7.6).

Proof. Given $\mathcal{H}^{*}$ we obtain

$$
\partial: \mathcal{H}_{1}^{*} \rightarrow \mathcal{H}_{0}^{*} \oplus k=A
$$

where $A$ is a $\tau$-commutative $k$-algebra by the multiplication $\mu$ in (5.5)(1). Moreover $\mathcal{H}_{1}^{*}=V$ is an $A$-module and one now readily checks by (4.2) and (1.6) that $\partial$ satisfies the properties of a $\tau$-crossed module. Conversely given $(\partial, \widetilde{\Gamma})$ as in (7.6) we obtain the secondary module $\mathcal{H}^{n}$ over $R_{n}$ by the commutative diagram (see (1.6))

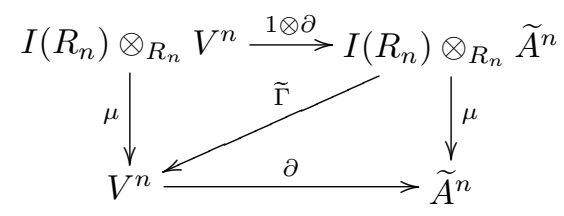

which we deduce from the diagram in (7.6). Moreover we define

$$
\mu: \mathcal{H}^{n} \otimes \mathcal{H}^{m} \rightarrow \mathcal{H}^{n+m}
$$

by the multiplication

$$
\widetilde{A}^{n} \otimes \widetilde{A}^{m}=\mathcal{H}_{0}^{n} \otimes \mathcal{H}_{0}^{m} \stackrel{\mu}{\longrightarrow} \widetilde{A}^{n+m}=\mathcal{H}_{0}^{n+m}
$$

of the algebra $\widetilde{A}$ over $R_{*}$ and by the map

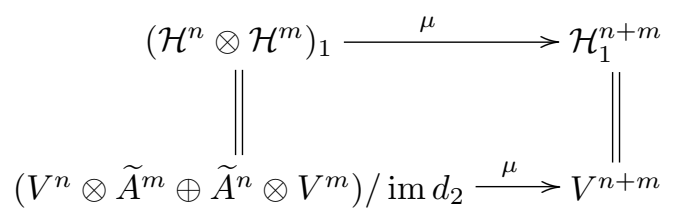

with $\mu(x \otimes a)=x \cdot a$ and $\mu(b \otimes y)=b \cdot y$ where $x \cdot a=\tau_{a, x} a \cdot x$. By (7.6)(1) this map is trivial on $\operatorname{im}\left(d_{2}\right)$. Now it is easy to show that the multiplication $\mu$ on $\mathcal{H}^{*}$ is associative (compare the proof on (7.4)) and $\tau$-commutative. Finally we have to check that $\mu$ on $\mathcal{H}^{*}$ is compatible with the equation in (4.2). This follows from (7.8)(2),(3) since for $\alpha^{\prime}=\alpha+\alpha_{k} \in I\left(R^{n}\right) \oplus k=R^{n}$ and $\beta^{\prime}=\beta+\beta_{k} \in I\left(R^{m}\right) \oplus k=$ $R^{m}$ we have

$$
\mu \Gamma_{\otimes}\left(\alpha^{\prime} \otimes \beta^{\prime} \otimes x \otimes y\right)=\widetilde{\Gamma}(\alpha \odot \beta \otimes x \cdot y)+\beta_{k} \widetilde{\Gamma}(\alpha \odot 1 \otimes x \cdot y)+\alpha_{k} \widetilde{\Gamma}(1 \odot \beta \otimes x \cdot y)
$$


Here we have $\alpha \odot \beta=(\alpha \odot 1)(1 \odot \beta)$ and hence in $I\left(R^{n+m}\right) \otimes_{R^{n+m}} V^{n+m}$ we have

$$
\begin{aligned}
\alpha \odot \beta \otimes x \cdot y & =(\alpha \odot 1)(1 \odot \beta) \otimes x \cdot y \\
& =\alpha \odot 1 \otimes(1 \odot \beta) x \cdot y \\
& =\alpha \odot 1 \otimes x \cdot \beta y
\end{aligned}
$$

Therefore we get by $(7.8)(2),(3)$

$$
\begin{aligned}
\mu \Gamma_{\otimes}\left(\alpha^{\prime} \otimes \beta^{\prime} \otimes x \otimes y\right) & =\widetilde{\Gamma}(\alpha \otimes x) \cdot\left(\beta y+\beta_{k} y\right)+\alpha_{k} x \cdot \widetilde{\Gamma}(\beta \otimes y) \\
& =\mu\left(\Gamma\left(\alpha^{\prime} \otimes x\right) \otimes \beta^{\prime} y+\left(\varepsilon\left(\alpha^{\prime}\right) x\right) \otimes \Gamma\left(\beta^{\prime} \otimes y\right)\right)
\end{aligned}
$$

Hence $\mu$ is compatible with the equation in (4.2).

We now use $\tau$-crossed modules (or by (7.10) equivalently $\tau$-commutative secondary algebras) for the following definition of symmetric cohomology which is a symmetric analogue of Hochschild cohomology in (6.3).

Definition 7.11. Let $R_{*}$ be a coefficient algebra with interchange elements $\tau$ for example let $R_{*}=k\left[\sigma_{*}\right]$ be the symmetric coefficient algebra. Let $H$ be a commutative graded $k$-algebra and let $M$ be an $H$-module. A $\tau$-crossed extension $\mathcal{E}$ of $H$ by $M$ is an exact sequence of graded $k$-vector spaces

$$
\mathcal{E}: 0 \longrightarrow M \longrightarrow V \stackrel{\partial}{\longrightarrow} A \stackrel{q}{\longrightarrow} H \longrightarrow 0
$$

Here $\partial$ is a $\tau$-crossed module as in (7.6). Moreover all maps are $A$-module morphisms with the $A$-module structure induced by the algebra map $q: A \rightarrow H$. A weak equivalence between two such $\tau$-crossed extensions is a commutative diagram

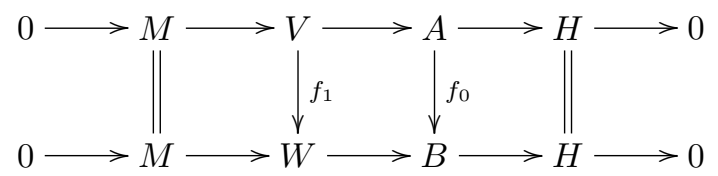

Here $f_{0}$ is a morphism of $k$-algebras over $R_{*}$ and $f_{1}$ is a $f_{0}$-equivariant homomorphism such that $\left(f_{0}, f_{1}\right)$ is compatible with $\widetilde{\Gamma}$. Let $\mathrm{SH}^{3}(H, M)$ be the set of weak equivalence classes of $\tau$-crossed extensions of $H$ by $M$. Below we show that $\mathrm{SH}^{3}(H, M)$ is a well defined set with the structure of a $k$-vector space.

homomorphism

At this moment we do not know a "cohomology theory" for commutative graded algebras $H$ which yields the cohomology $\mathrm{SH}^{3}(H, M)$ above.

We have the canonical natural homomorphism

$$
\mathrm{SH}^{3}(H, M) \rightarrow \mathrm{HH}^{3}(H, M)
$$

which carries the weak equivalence class of the $\tau$-crossed extension $\mathcal{E}$ to the weak equivalence class of the underlying crossed extension $\mathcal{E}_{(k)}$ given by (7.8), (7.4) and (7.5). We need the following "free" objects.

Definition 7.13. Let $V$ be a graded $k$-vector space. Then the free $R_{*}-$ module $R_{*} \odot V$ generated by $V$ is given by

$$
\left(R_{*} \odot V\right)^{n}=R_{n} \otimes V^{n} .
$$


For an $R_{*}$-module $W$ we obtain the tensor algebra over $R_{*}$ by

$$
\bar{T}(W)=\bigoplus_{n \geqslant 0} W^{\bar{\otimes} n}
$$

where $W^{\bar{\otimes} 0}=k$ and $W^{\bar{\otimes} n}$ is the $n$-fold $\bar{\otimes}$-product $W \bar{\otimes} \ldots \bar{\otimes} W$ defined in (7.1). For the tensor algebra $T(V)$ over $k$ we get

$$
\bar{T}\left(R_{*} \odot V\right)=R_{*} \odot T(V)
$$

so that $R_{*} \odot T(V)$ is the free $k$-algebra over $R_{*}$ generated by $V$ with the multiplication

$$
(\alpha \otimes x) \cdot(\beta \otimes y)=\alpha \odot \beta \otimes x \cdot y
$$

for $\alpha, \beta \in R_{*}$ and $x, y \in T(V)$. Let

$$
K_{\tau} \subset R_{*} \odot T(V)=A
$$

be the $R_{*}$-submodule generated by elements $1 \otimes y \cdot x-\tau_{x, y} \otimes x \cdot y$ for $x, y \in T(V)$. Then $K_{\tau}$ generates the ideal $A \cdot K_{\tau} \cdot A$ and the $R_{*}$-quotient module

$$
\Lambda=A / A \cdot K_{\tau} \cdot A
$$

is the free $\tau$-commutative $k$-algebra over $R_{*}$.

Given a $\tau$-commutative $k$-algebra $A$ over $R_{*}$ and a $k$-linear map $d: V \rightarrow A$ with $\varepsilon d=0$ we obtain the following push out diagram in the category of $R_{*}$-modules

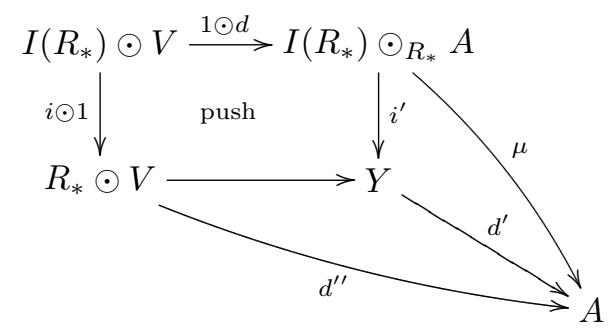

Here $d^{\prime \prime}$ is defined by $d^{\prime \prime}(\alpha \otimes x)=\alpha \cdot d(x)$. The pair $\left(\mu, d^{\prime \prime}\right)$ induces the $R_{*}$-linear map $d^{\prime}$ which thus determines the map of $A$-modules $\partial^{\prime}$ and $\partial$ in the following commutative diagram

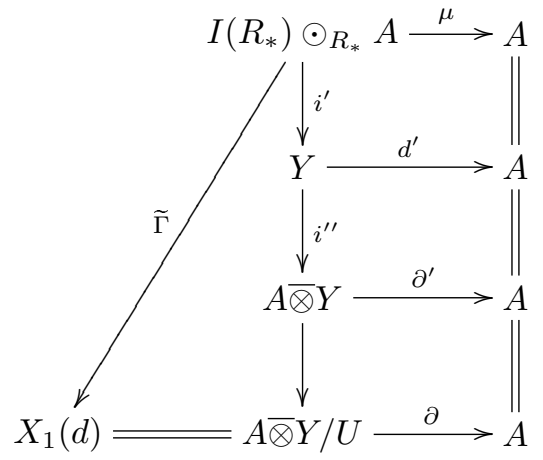


with $\widetilde{\widetilde{\Gamma}}=i^{\prime \prime} i^{\prime}$ and $\widetilde{\Gamma}=p \widetilde{\widetilde{\Gamma}}=p i^{\prime \prime} i^{\prime}$. Here $i^{\prime \prime}$ is defined by $i^{\prime \prime}(y)=1 \otimes y$ and $\partial^{\prime}$ is defined by $\partial^{\prime}(a \otimes y)=a \cdot d^{\prime}(y)$. Let $U$ be the $A$-submodule of $A \bar{\otimes} Y$ generated by the elements

$$
\begin{gathered}
\left(\partial^{\prime} x\right) \cdot y-\tau_{\partial^{\prime} y, x}\left(\partial^{\prime} y\right) \cdot x, \\
a \cdot \widetilde{\widetilde{\Gamma}}(\beta \otimes b)-\widetilde{\widetilde{\Gamma}}(1 \odot \beta \otimes a \cdot b),
\end{gathered}
$$

with $x, y \in A \bar{\otimes} Y$ and $a, b \in A, \beta \in I\left(R_{*}\right)$. One readily checks that $U$ is in the kernel of $\partial^{\prime}$ so that $\partial^{\prime}$ induces the $A$-module map $\partial$ on the quotient $X_{1}(d)=A \bar{\otimes} Y / U$. We claim that $(\partial, \widetilde{\Gamma})$ is a well define $\tau$-crossed module which is the free $\tau$-crossed module with basis $(V, d)$.

Finally we define for a commutative graded $k$-algebra the free $H$-module with basis $V$ by $H \otimes V$.

Definition 7.14. Let $H$ be a commutative graded $k$-algebra and consider a long exact sequence

$$
\mathcal{R}: \ldots \longrightarrow C_{2} \longrightarrow C_{1} \stackrel{\partial}{\longrightarrow} \Lambda \stackrel{q}{\longrightarrow} H \stackrel{0}{\longrightarrow}
$$

Here $\Lambda$ is a free $\tau$-commutative $k$-algebra over $R_{*}$ and $(\partial, \widetilde{\Gamma})$ is a free $\tau$-crossed module and $C_{i}, i \geqslant 2$, is a free $H$-module. All maps in the sequence are $\Lambda$-module morphisms where $C_{i}$ and $H$ are $\Lambda$-modules via the algebra map $q$. Then we call $\mathcal{R}$ a free $\tau$-crossed resolution of $H$.

It is easy to see that free $\tau$-crossed resolutions exist. Moreover given a $\tau$-crossed extension $\mathcal{E}$ as in (7.11) we can choose a commutative diagram $(n \geqslant 2)$

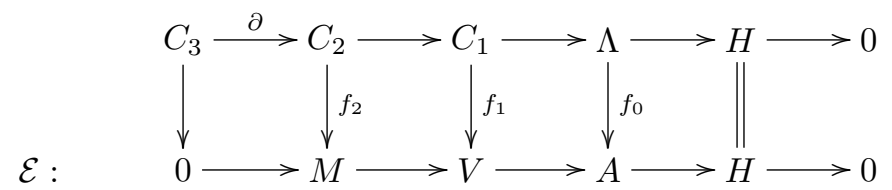

so that one gets a weak equivalence

$$
\left(f_{2}\right)_{*} \mathcal{R}_{2} \rightarrow \mathcal{E}
$$

where $\mathcal{R}_{2}$ is the $\tau$-crossed extension

$$
0 \rightarrow C_{2} / \partial C_{3} \rightarrow C_{1} \rightarrow \Lambda \rightarrow H \rightarrow 0
$$

given by $\mathcal{R}$. This implies that $\mathrm{SH}^{3}(H, M)$ is actually a set. In fact, the function

$$
\psi: \operatorname{Hom}_{H}\left(C_{2} / \partial C_{3}, M\right) \rightarrow \mathrm{SH}^{3}(H, M)
$$

which carries $f_{2}: C_{2} / \partial C_{3} \rightarrow M$ to the weak equivalence class of $\left(f_{2}\right)_{*} \mathcal{R}_{2}$ is surjective. Using this surjection it is possible to define the $k$-vector space structure of $\mathrm{SH}^{3}(H, M)$.

\section{Appendix: Eilenberg-MacLane spaces}

Let $k$ be a commutative ring, for example a field. For the definition of $Z^{n}=$ $K(k, n)$ in $(2.5)$ we shall need the following categories and functors; compare Goerss- 
Jardine $[\mathbf{G J}]$. Let Set and Mod be the category of sets and $k$-modules respectively and let $\Delta$ Set and $\Delta$ Mod be the corresponding categories of simplicial objects in Set and Mod respectively. We have functors

$$
\text { Top* } \stackrel{\text { Sing }}{\longrightarrow}(\Delta \text { Set })^{*} \stackrel{\mid 1}{\longrightarrow} \text { Top }^{*}
$$

given by the singular set functor Sing and the realization functor $\mid$. Moreover we have

$$
\Delta \text { Set } \stackrel{R}{\longrightarrow} \Delta \operatorname{Mod} \stackrel{\Phi}{\longrightarrow}(\Delta \text { Set })^{*}
$$

where $k$ carries the simplicial set $X$ to the free $k$-module generated by $X$ and where $\Phi$ is the forgetful functor which carries the simplicial module $A$ to the underlying simplicial set. Moreover we need the Dold-Kan functors

$$
\mathbf{C h}_{+} \stackrel{\Gamma}{\longrightarrow} \Delta \operatorname{Mod} \stackrel{N}{\longrightarrow} \mathbf{C h}_{+}
$$

where $\mathbf{C h}_{+}$is the category of chain complexes in Mod concentrated in degree $\geqslant 0$. Here $N$ is the normalization functor which by the Dold-Kan theorem is an equivalence of categories with inverse $\Gamma$. For a pointed space $V$ let

$$
K(V)=|\Phi S(V)| \text { with } S(V)=\frac{k \operatorname{Sing}(V)}{k \operatorname{Sing}(*)}
$$

Hence $K:$ Top $^{*} \rightarrow$ Top$^{*}$ carries a pointed space to a topological $k$-module. We define the binatural map

$$
\bar{\otimes}: K(V) \times K(W) \rightarrow K(V \wedge W)
$$

as follows. We have

$$
\operatorname{Sing}(V \times W)=\operatorname{Sing}(V) \times \operatorname{Sing}(W)
$$

and this bijection induces a commutative diagram in $\Delta \mathrm{Mod}$

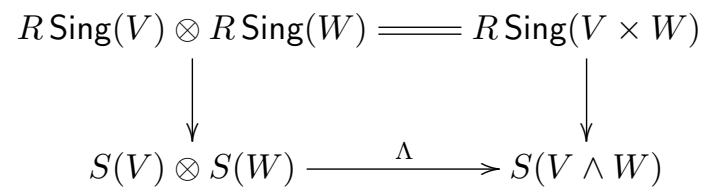

The vertical arrows are induced by quotient maps. For $k$-modules $A, B$ let $\otimes$ : $A \times B \rightarrow A \otimes_{k} B$ be the map in Set which carries $(a, b)$ to the tensor product $a \otimes b$. Of course this map $\otimes$ is bilinear. Moreover for $A, B$ in $\Delta$ Mod the map $\otimes$ induces the map $\otimes: \Phi(A \times B) \rightarrow \Phi(A \otimes B)$ in Set and the realization functor yields

$$
|\otimes|:|\Phi A| \times|\Phi B|=|\Phi(A \times B)| \rightarrow|\Phi(A \otimes B)|
$$

Hence for $A=S(V)$ and $B=S(W)$ we get the composite

$$
|\Phi S(V)| \times|\Phi S(W)| \stackrel{|\otimes|}{\longrightarrow}|\Phi(S(V) \otimes S(W))| \stackrel{|\Phi \Lambda|}{\longrightarrow}|\Phi S(V \wedge W)|
$$

and this is the map $\bar{\otimes}$ above. One readily checks that $\bar{\otimes}$ is bilinear with respect to the topological $k$-module structure of $K(V), K(W)$ and $K(V \wedge W)$ respectively. 
Moreover the following diagram commutes

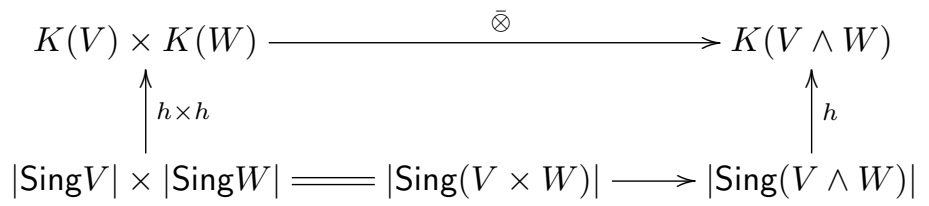

Here the Hurewicz map $h$ is the realization of the map in $\Delta$ Set

$$
\operatorname{Sing}(V) \rightarrow \Phi k \operatorname{Sing}(V) \rightarrow \Phi S(V)
$$

which carries an element $x$ in $\operatorname{Sing}(V)$ to the corresponding generator in $k \operatorname{Sing}(V)$.

Let $S^{n}=S^{1} \wedge \ldots \wedge S^{1}$ be the $n$-fold smash product of the 1 -sphere $S^{1}$. Then the symmetric group $\sigma_{n}$ acts on $S^{n}$ by permuting the factors $S^{1}$. It is well known that this action of $\sigma_{n}$ on $S^{n}$ induces the sign-action of $\sigma_{n}$ on the homology $H_{n}\left(S^{n}\right)=k$. We define the Eilenberg-MacLane space $Z^{n}$ by

$$
Z^{n}=K\left(S^{n}\right)=\left|\Phi \frac{k \operatorname{Sing}\left(S^{n}\right)}{k \operatorname{Sing}(*)}\right|
$$

Since $K$ is a functor we see that $\sigma_{n}$ also acts on $K\left(S^{n}\right)$ via $k$-linear automorphisms. We define the multiplication map $\mu_{m, n}$ by

$$
\mu: Z^{m} \times Z^{n}=K\left(S^{m}\right) \times K\left(S^{n}\right) \stackrel{\bar{\otimes}}{\longrightarrow} K\left(S^{m} \wedge S^{n}\right)=Z^{m+n}
$$

where $S^{m} \wedge S^{n}=S^{m+n}$ and where we use $\left(^{*}\right)$. Diagram (**) implies that $\mu$ induces the cup product in cohomology. The Eilenberg-MacLane spaces $Z^{n}$ together with the multiplication map $\mu$ satisfy the axioms of Karoubi $[\mathbf{K a}]$.

\section{References}

[B] Baues, H.--J.: The algebra of secondary cohomology operations, chapter 6, Preprint 2001.

[BD] Berrick, A.--J. and Davydov, A. A.: Splitting of Gysin extensions. Preprint (1999) group. K-theory 3 (1989) 307-338.

[BM] Baues, H.--J. and Minian, E. G.: Crossed extensions of algebras and Hochschild cohomology. To appear in this proceedings.

[GJ] Goerss, P. and Jardine, J. F.: Simplicial homotopy theory. Birkhäuser Verlag, Progress in Math. 174, (1999) 510 pages

[H] Huebschmann, J.: Crossed $n$-fold extensions of groups and cohomology. Comment. Math. Helvetici 55 (1980) 302-314

[HSS] Hovey, M. and Shipley, B. and Smith, J.: Symmetric spectra. Journal of the AMS 13 (2000) 149-208

[K] Kristensen, L.: On secondary cohomology operations, Math. Scand. 12 (1963) 57-82

[L] Loday, J.-L.: Cyclic Homology, Springer Verlag (1992) 
[Lu] Lue, S.-T.: Cohomology of algebras relative to a variety. Math. Z. 121 (1971) 220-232

[Ka] Karoubi, M.: Formes differentielle non commutatives et operation de Steenrod. Topology 34 (1995) 699-715

[P] Porter, T.: Extensions, crossed modules and internal categories in categories of groups with operations. Proc. Edinburgh Mayh. Soc. (2) 30 (1987) $373-381$

[St] Stover, C. R.: The equivalence of certain categories of twisted Lie and Hopf algebras over a commutative ring. Journal of pure and applied Algebra 86 (1993) 289-326

[W] Whitehead, J. H. C.: Combinatorial homotopy II, Bull. AMS 55 (1949) 213-245

This article may be accessed via WWW at http://www.rmi.acnet.ge/hha/ or by anonymous ftp at

ftp://ftp.rmi.acnet.ge/pub/hha/volumes/2002/n2a2/v4n2a2.(dvi,ps,pdf)

Hans-Joachim Baues baues@mpim-bonn.mpg.de

Max-Planck-Institut für Mathematik

Vivatsgasse 7

D-53111 Bonn

Germany 